Algebraic $\&$ Geometric Topology

Volume 3 (2003) 921-968

Published: 2 October 2003

ATG

\title{
Algebraic linking numbers of knots in 3-manifolds
}

\author{
RoB SCHNEIDERMAN
}

\begin{abstract}
Relative self-linking and linking "numbers" for pairs of oriented knots and 2-component links in oriented 3-manifolds are defined in terms of intersection invariants of immersed surfaces in 4-manifolds. The resulting concordance invariants generalize the usual homological notion of linking by taking into account the fundamental group of the ambient manifold and often map onto infinitely generated groups. The knot invariants generalize the type 1 invariants of Kirk and Livingston and when taken with respect to certain preferred knots, called spherical knots, relative self-linking numbers are characterized geometrically as the complete obstruction to the existence of a singular concordance which has all singularities paired by Whitney disks. This geometric equivalence relation, called $W$-equivalence, is also related to finite type 1-equivalence (in the sense of Habiro and Goussarov) via the work of Conant and Teichner and represents a "first order" improvement to an arbitrary singular concordance. For null-homotopic knots, a slightly weaker equivalence relation is shown to admit a group structure.
\end{abstract}

AMS Classification 57M27; 57N10, 57M25

Keywords Concordance invariant, knots, linking number, 3-manifold

\section{Introduction}

Working in the setting of finite type invariants, Kirk and Livingston defined families of type 1 invariants for knots in many 3 -manifolds and described indeterminacies that may arise when one attempts to define relative invariants for homotopically essential pairs of knots [19, 20]. The invariants of Kirk and Livingston are extracted from the homology classes of double-point loops corresponding to crossing changes in the knots during a homotopy. This paper will extract invariants from the homotopy classes of such double-point loops. The choices involved in identifying free homotopy classes with elements in the fundamental group of the ambient manifold require keeping careful track of conjugation actions and the resulting linking "numbers" take values in a target orbit space which is in general just a set (with a well-defined zero element) and depends in a rather subtle way on the knots and manifolds being considered. 
Some of the benefits justifying this somewhat unusual target include extending the Kirk-Livingston invariants non-trivially to many more manifolds (e.g. integral homology spheres) and showing they are concordance (not just isotopy) invariants, as well as providing a clear geometric characterization of the invariants in terms of Whitney disks and demonstrating computability of the indeterminacies.

The combination of 3- and 4-dimensional methods used in [19] and [20] highlights the suggestive relation between the various crossing change diagrams of 3-dimensional finite type theory and cross-sections of generic singularities of surfaces in 4-dimensions. The central idea of this paper is that this relation can be further exploited by applying (a generalization of) Wall's quadratic intersection form to the trace of a homotopy of knots. This approach provides a connection between two advancing lines of research: the extensions of finite type theories to arbitrary 3-manifolds (e.g. [2, 9, 18, 19, 20] and many others) and the developing theory of Whitney towers which detect the failure of the Whitney move in dimension 4 (e.g. [3, 4, 22, 23, 26, 27]).

Knot theory in a non-simply connected manifold breaks naturally into the study of free homotopy classes of knots (or classes of 0 -equivalent knots in the language of finite type theory). We assume that our manifolds are oriented and equipped with basepoints. For each element $\gamma \in \pi_{1} M$ in the fundamental group of a 3manifold $M$, let $\mathcal{K}_{\gamma}(M)$ denote the set of oriented knots in the free homotopy class determined by (the conjugacy class of) $\gamma$ and let $\mathcal{C}_{\gamma}(M)$ denote the set $\mathcal{K}_{\gamma}(M)$ modulo concordance (details in 2.2). Thus, any two knots $k$ and $j$ in $\mathcal{K}_{\gamma}$ co-bound an immersed annulus in $M \times I$ ( $I$ the unit interval) and our invariants will (1) provide obstructions to $k$ and $j$ representing the same element in $\mathcal{C}_{\gamma}(M)$, that is, obstructions to $k$ and $j$ co-bounding an embedded annulus in $M \times I$, and (2) provide geometric information towards improving a singular concordance in the sense that all singularities can be paired by Whitney disks.

Although we will not work explicitly in the setting of finite type invariants, the reader familiar with the type 1 invariants of Kirk and Livingston will notice that they factor through the invariants defined here (with their chosen cohomology class corresponding to a representation from $\pi_{1} M$ to a cyclic group).

A survey of our main results follows.

Null-homotopic knots Our first observation (details in Section 3) is that, for any (oriented null-homotopic) knot $k \in \mathcal{K}_{1}(M)$, Wall's self-intersection 
invariant $\mu$ (which counts signed double-point loops) can be applied to the trace of any null-homotopy of $k$ in $M \times I$, yielding an algebraic self-linking number $\mu(k)$ which takes values in a quotient $\widetilde{\Lambda}$ of the free abelian group $\Lambda:=\mathbb{Z}\left[\pi_{1} M\right]$ generated by the elements of $\pi_{1} M$. The quotient is by two relations: The inversion relation $g=g^{-1}$ for group elements corresponds to changing the choice of orientation of a double-point loop, and the cusp relation $1=0$ corresponds to the fact that local cusp homotopies create or eliminate double point loops having the trivial group element $1 \in \pi_{1} X$ (we are working with unframed knots). The concordance invariance of self-linking numbers is a direct consequence of the 3-dimensional Sphere Theorem (see Lemma 3.2.1) and by clasp-doubling embedded loops it is not hard to construct knots realizing all elements in $\widetilde{\Lambda}$; this is the content of the following theorem which is proved in Section 3.

Theorem 1 The map $k \mapsto \mu(k)$ induces a well-defined map from $\mathcal{C}_{1}(M)$ onto $\widetilde{\Lambda}$.

Due to the choice of basing of $k$, the image of $\mu(k) \in \widetilde{\Lambda}$ is only well-defined up to conjugation by elements of $\pi_{1} M$; however, there are no further indeterminacies and if $M$ is not simply connected then self-linking numbers detect many nullhomotopic knots which are not null-concordant, i.e., which do not bound an embedded 2-disk in $M \times I$. For example, if a knot $k_{g} \in M$ is constructed as the clasped-double of a null homologous loop which represents any non-trivial element $g$ in the commutator subgroup of $\pi_{1} M$, then $\mu\left(k_{g}\right)= \pm g \neq 0 \in \widetilde{\Lambda}$ (Figure 4 in 3.2) showing that $k_{g}$ is not null-concordant and not 1-equivalent to the unknot (see paragraph after Theorem 3 below), facts not detected by the homological invariants of [19] and [20].

Essential knots In Section 4 we will define relative algebraic self-linking numbers for pairs of essential knots, i.e. knots in $\mathcal{K}_{\gamma}(M)$ for $\gamma \neq 1$, by applying a generalization of Wall's self-intersection invariant to immersed annuli in $M \times I$. This generalized $\mu$-invariant for immersed annuli naturally takes values in an abelian group $\tilde{\Lambda}_{\gamma}$ generated by the double cosets of the fundamental group of the ambient manifold by the cyclic subgroup $\langle\gamma\rangle$ generated by the annulus; in our case, $\gamma$ will be a knot longitude. If $k$ and $j$ are knots in $\mathcal{K}_{\gamma}(M)$ then the relative self-linking number $\mu_{k}(j)$ of $j$ with respect to $k$ takes values in an orbit space of $\tilde{\Lambda}_{\gamma}$ under two group actions: There is a conjugation action by the centralizer $\zeta(\gamma)$ which corresponds to basing choices and a more subtle action of a certain indeterminacy sub-group

$$
\Phi(k) \leq \tilde{\Lambda}_{\gamma} \rtimes \zeta(\gamma)
$$


which accounts for the effect that pre-composing by a singular self-concordance of $k$ has on $\mu_{k}(j)$.

The following example illustrates some basic properties of relative self-linking numbers. More subtle properties will be exhibited in Section 8 .

Example Illustrated in Figure 1 are two knots $k$ and $j$ in $\mathcal{K}_{x y z}(M)$ where $M$ is the product $F \times S^{1}$ of a thrice punctured 2-disk $F$ with the circle and $\pi_{1} M=\langle x, y, z\rangle \times\langle t\rangle$ is the cartesian product of the free group on $x, y$ and $z$ (represented by loops in $F$ around the punctures) with the central cyclic group generated by the element $t$ (represented by a circle factor of $M$ ). The figure shows $M$ cut open along $F$ cross a point.

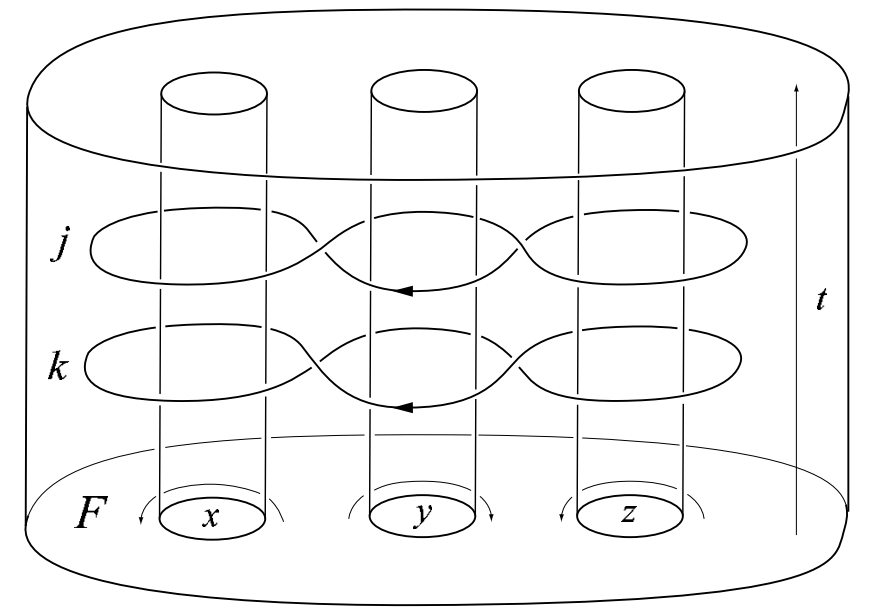

Figure 1

In this case, with respect to $k$, both the conjugacy and indeterminacy subgroup actions are trivial (see Example 5.4.2) so the relative self-linking number $\mu_{k}(j)$ takes values in the group $\widetilde{\Lambda}_{x y z}$. The two obvious crossing changes that change $k$ into $j$ describe the trace $H$ of a homotopy with two singularities of differing sign. The group elements associated to these singularities can be computed from the figure yielding (up to a sign from global orientation conventions) $\mu_{k}(j)=$ $\mu(H)=x-x y$ which is non-zero in $\widetilde{\Lambda}_{x y z}$. That $k$ and $j$ are not isotopic can also, in this case, be detected by the Kirk-Livingston invariants (using a cohomology class that is non-trivial on $y$ ); as a result of the next theorem we see, furthermore, that $k$ and $j$ are not concordant. 
Although the target for $\mu_{k}(j)$, denoted $\tilde{\Lambda}_{\gamma} /(\Phi(k), \zeta(\gamma))$, does not in general have a group structure (Remark 4.7.2), it does have a well-defined orbit of $0 \in \tilde{\Lambda}_{\gamma}$ and so it makes sense to speak of $\mu_{k}(j)$ vanishing. The following theorem will be proved in Section 4 .

Theorem 2 For each $k \in \mathcal{K}_{\gamma}(M)$, the map $j \mapsto \mu_{k}(j)$ vanishes on knots concordant to $k$ and induces a well-defined map

$$
\mathcal{C}_{\gamma}(M) \rightarrow \tilde{\Lambda}_{\gamma} /(\Phi(k), \zeta(\gamma))
$$

onto the target.

Thus, the target reflects some of the structure of $\mathcal{C}_{\gamma}(M)$ and a variety of properties which can occur are illustrated in the examples of Section 8 (including torsion (8.1)). Describing this target depends to a large extent on understanding the indeterminacy sub-group $\Phi(k)$. A satisfying theme that will emerge is that these indeterminacies, which come from essential tori and 2-spheres in $M$, can be measured in terms of intersections between lower-dimensional manifolds. A generating set for $\Phi(k)$ is given in Proposition 5.4.1.

The indeterminacies due to 2 -spheres are computed in terms of an intersection pairing $\widetilde{\lambda}(\sigma, k)$ between $\pi_{2}(M)$ and $\mathcal{K}_{\gamma}(M)$ (5.1). This allows our knot invariants to be defined in reducible 3 -manifolds, which is desirable even if one is ultimately interested in the manifold structure; for instance, surgering a knot in a reducible manifold can yield an irreducible one.

The indeterminacies due to tori correspond to singular self-concordances which project to essential immersed tori in $M$ and only exist if the centralizer $\zeta(\gamma)$ of $\gamma$ is non-cyclic. In this case, the computation of $\Phi(k)$ can be reduced to a computation in a Seifert fibered submanifold that carries $\zeta(\gamma)$ using the 3manifold structure theorems of Jaco-Shalen [15, 16], Johannson [17], Gabai [8] and Casson-Jungreis [1]. This follows the approach used by Kalfagianni [18] to show the existence of finite type knot invariants in many irreducible 3-manifolds. These indeterminacies correspond to intersections between knots and tori in $M$ and can in general be computed in terms of an intersection pairing between curves in an orbit surface of a Seifert fibered sub-manifold of $M$ ([24]). In this paper we will mostly be concerned with cases where these indeterminacies are trivial.

Spherical knots It turns out that there exist many knots for which the indeterminacy subgroup $\Phi(k)$ is completely determined by the pairing $\widetilde{\lambda}(\sigma, k)$ 
between the knots and 2-spheres; these knots, called spherical knots (6.1), play a preferred role (analogous to the unknot in the trivial homotopy class) in the geometric interpretation of linking invariants:

Theorem 3 If $k \in \mathcal{K}_{\gamma}(M)$ is spherical, then for any knots $j$ and $j^{\prime}$ in $\mathcal{K}_{\gamma}(M)$ the following are equivalent:

(i) $\mu_{k}(j)=\mu_{k}\left(j^{\prime}\right)$.

(ii) There exists a singular concordance between $j$ and $j^{\prime}$ such that all singularities are paired by Whitney disks.

As illustrated later in Example 8.2, there are examples where $\mu_{k}(j)=\mu_{k}\left(j^{\prime}\right)$ for a non-spherical knot $k$ and property (ii) of Theorem 3 does not hold (as detected by $\mu_{k_{0}}(j) \neq \mu_{k_{0}}\left(j^{\prime}\right)$ for some spherical $\left.k_{0}\right)$.

$W$-equivalence Theorem 3 shows that, with respect to spherical knots, relative self-linking numbers inherit the geometric characterization of Wall's selfintersection invariant in terms of Whitney disks (see Proposition 2.1.3). The equivalence relation on $K_{\gamma}(M)$ defined by property (ii) of Theorem 3 is called $W$-equivalence and represents a "first order" improvement over an arbitrary singular concordance (a 0-equivalence) in the following sense: Conant and Teichner have characterized finite type $n$-equivalence (in the sense of Habiro and Goussarov) of knots in 3-manifolds in terms of the notion of 3-dimensional capped grope cobordism $[5,6]$. In their language, 1-equivalence corresponds to capped surface cobordism. Such a capped surface bordism in $M$ can be pushed into $M \times I$ and surgered to a $W$-equivalence. (See [22] for details including higher orders.) Thus, relative self-linking numbers give obstructions to 1-equivalence of knots.

Example In the example illustrated in Figure 1 above, the knot $k$ is spherical (see examples 5.4.2 and 6.1.2), hence $k$ and $j$ are not $W$-equivalent, by taking $j^{\prime}=k$ in Theorem 3 , since $\mu_{k}(j)-\mu_{k}(k)=\mu_{k}(j)$ is non-zero.

We are assured of the existence of spherical knots in all homotopy classes by restricting to a large class $\mathcal{M}$ of 3 -manifolds which do not contain circle bundles over non-orientable surfaces whose total spaces are orientable and do not contain certain Seifert fibered spaces containing non-vertical tori as sub-manifolds (see Section 7). 
Theorem 4 For any $M \in \mathcal{M}$ and any $\gamma \in \pi_{1} M$, there exists a spherical knot $k_{0}$ in $\mathcal{K}_{\gamma}(M)$.

Thus, relative self-linking numbers characterize $W$-equivalence in $\mathcal{M}$.

2-component links The discussion so far can be applied similarly to define (relative) concordance invariants for 2-component links in terms of (a generalization (9.3) of) Wall's intersection pairing $\lambda$ (2.1) with analogous results. This is sketched in Section 9. One result worth mentioning here is that the (absolute) algebraic linking number (9.1.1) for a 2-component link of null-homotopic knots plays a role in defining a group structure on a quotient of $K_{1}(M)$ (see 9.1.3).

Conventions For the most part, standard 3- and 4-dimensional techniques and terminology are used throughout. Irreducible 3-manifolds will be allowed to have spherical boundary components. The closed unit interval $[0,1]$ will be denoted by $I$ and occasionally be reparametrized implicitly. We work in the smooth oriented category with specific orientations usually suppressed.

Acknowledgments I am happy to thank Paul Kirk and Peter Teichner for helpful conversations, and my former advisor Rob Kirby for his guidance and support. Thanks also to the referee whose careful reading and thoughtful comments have significantly contributed to improving the exposition. This work was supported in part by an NSF Postdoctoral Fellowship and the Max-PlanckInstitut für Mathematik.

\section{Preliminaries}

This section briefly reviews the 4-dimensional version of Wall's intersection and self-intersection invariants, $\lambda$ and $\mu$, as well as the notions of concordance and singular concordance and also serves to fix notation. See also [7] for more details.

\subsection{Wall's intersection invariants}

Let $D$ and $E$ be properly immersed 2-spheres or 2-disks (rel $\partial$ ) in a 4-manifold $X$, that is, boundary is embedded in boundary and interior immersed in interior. 
After a small perturbation ( $\operatorname{rel} \partial$ ), $D$ and $E$ can be assumed to be in general position, so that $D$ meets $E$ in a finite set of transverse intersection points and each of $D$ and $E$ have finitely many transverse self-intersection points or double point singularities. Neighborhoods of an intersection point $p$ in $D$ and $E$ are called sheets of $D$ and $E$ (at $p$ ). Fix whiskers for each of $D$ and $E$; that is, choose an arc in $X$ connecting the basepoint of $X$ to a basepoint on $D$ and likewise for $E$.

\subsubsection{Intersection numbers}

Each point $p \in D \cap E$ determines an element $g_{p} \in \pi_{1} X$ from the following loop: First go along the whisker on $D$ from the basepoint of $X$ to the basepoint of $D$, then along $D$ (avoiding all double points) to $p$, then along $E$ (avoiding all double points) to the basepoint on $E$ and then back along $E$ 's whisker to the basepoint of $X$. Since $D$ and $E$ are simply connected, $g_{p}$ does not depend on how the loop runs between $p$ and the basepoints on $D$ and $E$. By summing (with appropriate signs) over all intersection points we get an intersection "number" in $\Lambda:=\mathbb{Z}\left[\pi_{1} X\right]$, the free abelian group generated by the elements of $\pi_{1} X$ :

Definition 2.1.1 The intersection number $\lambda(D, E)$ of $D$ and $E$ is defined by

$$
\lambda(D, E):=\sum(\operatorname{sign} p) \cdot g_{p} \in \Lambda
$$

where the sum is over all intersection points $p \in D \cap E$ and $\operatorname{sign} p$ equals +1 (resp. -1 ) if the orientation of $X$ at $p$ agrees (resp. disagrees) with the orientation determined by the sheets of $D$ and $E$ at $p$.

Note that the $g_{p}$ are all computed using the fixed whiskers on $D$ and $E$. Changing the whisker on $D$ (resp. $E$ ) changes $\lambda(D, E)$ by left (resp. right) multiplication by an element of $\pi_{1} X$.

\subsubsection{Self-intersection numbers}

For each double point $p$ of $D$, define $g_{p} \in \pi_{1} X$ from the following loop: First go along the whisker on $D$ from the basepoint of $X$ to the basepoint of $D$, then along $D$ (avoiding all double points) to $p$, then change sheets at $p$ and go back along $D$ (avoiding all double points) to the basepoint on $D$ and then back along $D$ 's whisker to the basepoint of $X$. Note that (for a fixed whisker) $g_{p}$ depends only on the choice of first sheet at $p$ and changing the order of 
sheet-change for the loop at $p$ changes $g_{p}$ to $g_{p}{ }^{-1}$. Note also that a local cusp homotopy (Figure 2) creates a double point $p$ with $g_{p}$ equal to the trivial element $1 \in \pi_{1} X$.

Definition 2.1.2 The self-intersection number $\mu(D)$ is defined by

$$
\mu(D):=\sum(\operatorname{sign} p) \cdot g_{p} \in \tilde{\Lambda}
$$

where the sum is over all double points $p$ of $D$ and $\operatorname{sign} p= \pm 1$ is determined by comparing the orientation of $X$ at $p$ with the orientation given by the two sheets of $D$ at $p$.

Here $\tilde{\Lambda}$ is as described in the introduction:

$$
\tilde{\Lambda}:=\frac{\Lambda}{\left\{g-g^{-1}\right\} \oplus \mathbb{Z}[1]}
$$

where $g$ ranges over $\pi_{1} X$ and $\mathbb{Z}[1]$ is generated by the trivial element $1 \in \pi_{1} X$. (This is a quotient as an abelian group, not as a ring.) Note that changing the whisker on $D$ changes $\mu(D)$ by conjugation by an element of $\pi_{1} X$.

Remark This definition of self-intersection number is sometimes referred to as the reduced self-intersection number; omitting the quotient by $\mathbb{Z}[1]$ yields an unreduced version which is only invariant under regular homotopy. Using the unreduced version would lead to an invariant of framed knots.

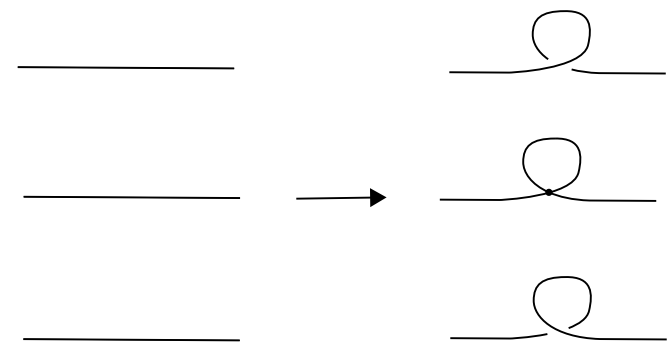

Figure 2: Before and after a local cusp homotopy of a surface in a 4-manifold

\subsubsection{Homotopy invariance and the geometry of Wall's invariant}

The homotopy invariance of intersection numbers can be shown by an analysis of the singularities of homotopies of surfaces ([7]): 
Proposition 2.1.3 In the above setting, $\lambda(D, E) \in \Lambda$ and $\mu(D) \in \tilde{\Lambda}$ depend only on the homotopy classes (rel $\partial$ ) of $D$ and $E$. The intersection number $\lambda(D, E)$ and self-intersection numbers $\mu(D)$ and $\mu(E)$ all vanish if and only if $D$ and $E$ are homotopic (rel $\partial$ ) to disjoint maps with all self-intersections paired by Whitney disks.

The disjointness property follows from standard manipulations of Whitney disks. (See 1.4 of [7] for an introduction to Whitney disks in 4-manifolds.) If the Whitney disks in the conclusion of Prop 2.1.3 happened to be disjointly embedded, correctly framed and with interiors disjoint from $D$ and $E$, then it would follow that $D$ and $E$ are homotopic (rel $\partial$ ) to disjoint embeddings.

Note that in a simply connected $4-$ manifold $\lambda(D, E)$ reduces to the usual homological intersection $D \cdot E \in \mathbb{Z}$ which counts the signed intersection points and that $\tilde{\Lambda} \cong 0$ so that $\mu$ always vanishes.

\subsection{Concordance and singular concordance}

Definition 2.2.1 Two knots $k$ and $j$ in a 3-manifold $M$ are concordant if there exists a properly embedded annulus $(C, \partial C) \hookrightarrow(M \times I, M \times \partial I)$ bounded by $k \subset M \times\{0\}$ and $j \subset M \times\{1\}$. The oriented boundary of the annulus $C$ is required to induce the difference of the orientations of the knots: $\partial C=k-j$. Such a $C$ is called a concordance between $k$ and $j$. Two links (collections of disjoint knots) $l$ and $l^{\prime}$ in $M$ are concordant if their components can be joined by a collection of properly embedded pairwise disjoint annuli in $M \times I$ where each annulus is a concordance between a component of $l$ and a component of $l^{\prime}$. The union of the annuli is a concordance between $l$ and $l^{\prime}$.

Concordance is clearly an equivalence relation on knots or links with a fixed number of components and isotopy is a special kind of level-preserving concordance. By allowing the annuli to be immersed we get the (much) weaker equivalence relation of singular concordance:

Definition 2.2.2 Two knots $k$ and $j$ in $M$ are singularly concordant if there exists a properly immersed annulus $(C, \partial C) \rightarrow(M \times I, M \times \partial I)$ bounded by $k \subset$ $M \times\{0\}$ and $j \subset M \times\{1\}$. Such a $C$ is called a singular concordance between $k$ and $j$ (or from $k$ to $j$ ). Two links $l$ and $l^{\prime}$ in $M$ are singularly concordant if their components can be joined by a collection of properly immersed annuli (not necessarily disjoint) in $M \times I$ where each annulus is a singular concordance between a component of $l$ and a component of $l^{\prime}$. 
The trace of a homotopy of knots is a (level-preserving) singular concordance and, by [11] and [10], the relations of singular concordance and homotopy are in fact equivalent for knots and links in 3-manifolds. It will be convenient to make statements in the a priori more general language of singular concordance, however explicit constructions will usually be described by homotopies.

\subsection{Whiskers for knots}

Recalling the notation in the introduction, we will use the fundamental group to index singular concordance classes of knots:

Definition 2.3.1 For each element $\gamma$ in the fundamental group of a 3-manifold $M$, let $\mathcal{K}_{\gamma}(M)$ denote the set of oriented knots (up to isotopy) in the free homotopy class determined by (the conjugacy class of) $\gamma$ and let $\mathcal{C}_{\gamma}(M)$ denote the set $\mathcal{K}_{\gamma}(M)$ modulo concordance.

This means that whenever we connect a knot $k \in \mathcal{K}_{\gamma}(M)$ by a whisker to the basepoint of $M$ we require that this basing satisfies $[k]=\gamma \in \pi_{1} M$.

\section{Null-homotopic knots}

This section contains the precise definition of the algebraic self-linking number $\mu(k)$ and the proof of Theorem 1. The arguments here also apply to algebraic linking numbers of 2-component links of null-homotopic knots as will be described in Section 9.

\subsection{Algebraic self-linking numbers}

Recall that our knots and manifolds are assumed oriented.

Definition 3.1.1 For $k \in \mathcal{K}_{1}(M)$ define the algebraic self-linking number $\mu(k)$ by

$$
\mu(k):=\mu(D) \in \widetilde{\Lambda}
$$

where $D$ is any properly immersed 2-disk in $M \times I$ bounded by $k \subset M \times\{0\}$ and $\mu(D)$ is Wall's self-intersection number as defined in 2.1.2. Here $\pi_{1}(M \times I)$ is identified with $\pi_{1} M$ via projection onto $M \times\{0\}$. The immersed 2-disk $D$ is a singular null-concordance of $k$ and is oriented by the orientation of $k$ (via some fixed convention). 
We may refer to $\mu(k)$ simply as a "self-linking number" for sake of brevity, since in the present context omitting "algebraic" should not cause confusion with the well known integer-valued self-linking number of a framed knot.

\subsection{Proof of Theorem 1}

Independence of choice of $D$ We show first that $\mu(k)$ does not depend on the choice of singular null-concordance. Let $D$ and $D^{\prime}$ be two singular nullconcordances of $k$. Then the union $S$ of $D$ and $D^{\prime}$ along $k$ (in two copies of $M \times I$ identified along $M)$ determines an element of $\pi_{2}(M \times I) \cong \pi_{2}(M)$ (see Figure 3).

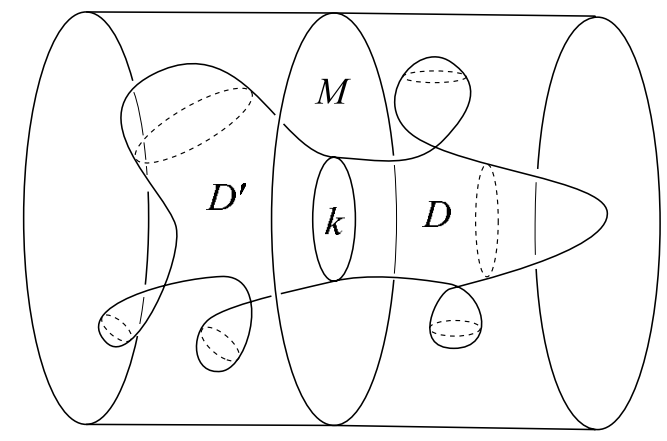

Figure 3

Lemma 3.2.1 Any $n$ elements of $\pi_{2}(M \times I)$ are represented by $n$ embedded pairwise disjoint 2-spheres.

Proof A well known consequence of the 3-dimensional Sphere Theorem is that $\pi_{2}(M)$ is generated as a module over $\pi_{1}(M)$ by disjoint embeddings (the 2-spheres that decompose $M$ into prime factors, together with any spherical boundary components and cross-sections of any $S^{2} \times S^{1}$ factors, see Proposition 3.12 of [13]). Tubing these generators together in $M \times I$ does not create any new intersections, so $\pi_{2}(M \times I)$ is spanned by disjoint embeddings.

Lemma 3.2.1 implies in particular that Wall's intersection form vanishes on $\pi_{2}(M \times I)$ and so

$$
\mu(S)=0=\mu(D)-\mu\left(D^{\prime}\right) .
$$

This shows that $\mu(k)$ does not depend on the choice of bounding disk. 
Concordance invariance If $C$ is a concordance from $k^{\prime}$ to $k$ then, up to conjugation, $\mu\left(k^{\prime}\right)=\mu(C \cup D)=\mu(D)=\mu(k)$ since $C$ has no singularities.

The map $k \mapsto \mu(k)$ is onto To construct a null-homotopic knot $k_{g} \subset M$ with $\mu\left(k_{g}\right)= \pm g$ for any element $g \in \pi_{1}(M)$, push an arc of a small circle around a loop representing $g$ and create a \pm -clasp with the circle (Figure 4). By iterating this procedure (or band summing together such clasps), one can realize anything in $\Lambda$ as $\mu(k)$ for some $k$.

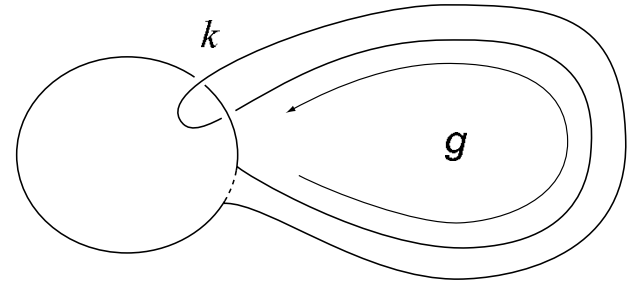

Figure 4: Creating a null-homotopic knot $k$ with $\mu(k)= \pm g$

Remark 3.2.2 Note that Lemma 3.2.1 implies that any homotopy invariant of 2 -spheres in a 4-manifold which vanishes on disjoint embeddings (and restricts to an invariant of immersed 2-disks rel boundary) will give a concordance invariant of null-homotopic knots in a 3 -manifold. In particular, the invariant $\tau$ of [26] can be used to define second order knot invariants whenever the first order invariants vanish (see [25]).

\section{Relative self-linking numbers}

This section introduces the definitions, conventions and notation necessary to define relative self-linking numbers $\mu_{k}(j)$ and gives the proof of Theorem 2 . Since basepoint issues will complicate the procedure, some motivation is in order. The goal is to construct a relative knot invariant that is as free from basepoint dependence as possible and has a clear geometric characterization. The first step (4.1) is to generalize Wall's $\mu$ homotopy invariant to immersed annuli in such a way that $\mu(A)$ vanishes if and only if the singularities of the immersed annulus $A$ can be paired up by Whitney disks (after a homotopy rel $\partial$ ). This is where the double coset space (by the cyclic subgroup $\langle\gamma\rangle$ ) enters the picture. Here also, the invariant in $\tilde{\Lambda}_{\gamma}$ is well-defined only up to a conjugation 
action by the centralizer subgroup $\zeta(\gamma)$, corresponding to the choice of whisker connecting the annulus to the basepoint of the ambient manifold.

Next, we want to apply $\mu$ to an immersed annulus joining two knots in $M \times$ I. (Such an immersed annulus will often be denoted $H$ since in practice it will usually be the trace of a homotopy between knots.) This requires fixing conventions (4.2) and more importantly examining the behavior of $\mu$ under composition of singular concordances, in particular singular self-concordances of a knot which may contribute indeterminacies to the final invariant. A subtle effect that arises is another conjugation action by $\zeta(\gamma)$ which comes from a loop traced out by a basepoint on the knot during a homotopy, together with the whiskers on the knots at either end of the homotopy; such a loop is called a latitude $(4.3,4.4)$ and this conjugation action prevents $\mu$ from being additive $(4.5,4.6)$. The effect of this non-additivity is captured by the action of an indeterminacy subgroup $\Phi(k)$ which depends in general on the "base" knot $k$ (4.7). This action is compatible with the basepoint conjugation action (4.7.2) and the precise definition of $\mu_{k}(j)$ is given in 4.8 .

Having successfully waded through these conventions and notations, the proof of Theorem 2 then more or less falls out of the definitions. In digesting the above conjugation actions, notations, etc., it may be helpful for the reader to draw schematic pictures along the lines of Figure 5 (in 4.9) in order to see the effect of compositions, whisker changes, etc. on the double-point loops counted by $\mu$.

\subsection{Self-intersection numbers for annuli}

Let $A:\left(S^{1} \times I, S^{1} \times\{0,1\}\right) \rightarrow(X, \partial X)$ be a properly immersed annulus in a 4 -manifold $X$. Choosing a whisker for $A$ identifies the image of $\pi_{1}\left(S^{1} \times I\right) \cong \mathbb{Z}$ with a cyclic subgroup $\langle\gamma\rangle$ of $\pi_{1} X$ generated by the image of a generating circle of the annulus. For each double point $p$ of $A$, associate an element $g_{p} \in \pi_{1} X$ from a loop in $A$ that changes sheets at $p$ (and avoids all other double points) together with the whisker on $A$. Note that $g_{p}$ is only well-defined up to left and right multiplication by powers of $\gamma$, corresponding to the loop in $A$ wandering around the circle direction before and after changing sheets at $p$. Denote by $\Lambda_{\gamma}$ the free abelian group additively generated by the double cosets of $\pi_{1} X$ by $\langle\gamma\rangle$, that is,

$$
\Lambda_{\gamma}:=\mathbb{Z}\left[\langle\gamma\rangle \backslash \pi_{1} X /\langle\gamma\rangle\right]=\frac{\mathbb{Z}\left[\pi_{1} X\right]}{\left\{g-\gamma^{n} g \gamma^{m}\right\}}
$$

where $n$ and $m$ range over the integers. 
To account for indeterminacies due to the choices of orientation of the doublepoint loops and from the introduction of trivial group elements via cusp homotopies, we take a further quotient and define

$$
\tilde{\Lambda}_{\gamma}:=\frac{\Lambda_{\gamma}}{\left\{g-g^{-1}\right\} \oplus \mathbb{Z}[1]}=\frac{\mathbb{Z}\left[\pi_{1} X\right]}{\left\{g-\gamma^{n} g^{ \pm 1} \gamma^{m}\right\} \oplus \mathbb{Z}[1]} .
$$

Definition 4.1.1 Let $A$ be a properly immersed annulus in a 4-manifold $X$ and $\langle\gamma\rangle$ be the image of the induced map on fundamental groups. Then the self-intersection number $\mu(A)$ of $A$ is defined by

$$
\mu(A):=\sum(\operatorname{sign} p) \cdot g_{p} \in \tilde{\Lambda}_{\gamma}
$$

where the sum is over all double points $p$ of $A$ and $\operatorname{sign} p$ comes from the orientations of $X$ and the sheets of $A$ at $p$ as usual.

Changing the whisker for $A$ in a manner that preserves the image $\langle\gamma\rangle$ of the fundamental group has the effect of conjugating $\mu(A)$ by an element of the centralizer subgroup $\zeta(\gamma):=\left\{g \in \pi_{1} X \mid g \gamma=\gamma g\right\} \leq \pi_{1} X$ (see 4.5 below). Thus, the orbit of $\mu(A)$ in $\tilde{\Lambda}_{\gamma}$ under conjugation by $\zeta(\gamma)$ is invariant.

In general, changing the whisker on $A$ changes both $\mu(A)$ and the target space by an isomorphism: $z \in \tilde{\Lambda}_{\gamma} \mapsto \alpha z \alpha^{-1} \in \tilde{\Lambda}_{\alpha \gamma \alpha^{-1}}$.

The same arguments as in the proof of Proposition 2.1.3 give:

Proposition 4.1.2 The above defined self-intersection number $\mu(A)$ is invariant under homotopy ( $\mathrm{rel} \partial$ ) and vanishes if and only if all singularities of $A$ can be paired by Whitney disks (perhaps after some local cusp homotopies).

\subsection{Conventions for singular concordances}

Let $H:\left(S^{1} \times I, S^{1} \times\{0,1\}\right) \rightarrow(M \times I, M \times\{0,1\})$ be a singular concordance between any knots $k$ and $j$ in $\mathcal{K}_{\gamma}(M)$. Orientations of $H$ and $M \times I$ are determined by the orientations on $k \subset M \times\{0\}$ and $M$ together with the orientation of $I$ so that $\partial H=k-j$. Identify $\pi_{1}(M \times I)$ with $\pi_{1} M$ via projection onto $M \times\{0\}$. Take a whisker for $k$ as the whisker for $H$. Since by convention (2.3) we only take whiskers for $k$ so that $[k]=\gamma$, the image of the fundamental group of the annulus equals $\langle\gamma\rangle \leq \pi_{1} M$.

Unless otherwise specified, these conventions will be assumed for all singular concordances. 


\subsection{Latitudes of singular concordances}

Let $H$ be a singular concordance from $k$ to $j$ with whiskers chosen so that $[k]=[j]=\gamma \in \pi_{1} M$. A latitude of $H$ is any arc that goes from the basepoint of $M$ in $M \times\{0\}$ along the whisker $w$ of $k$, then along $H$, then along the whisker $w^{\prime}$ of $j$ to the basepoint of $M$ in $M \times\{1\}$. The projection of a latitude of $H$ to $M \times\{0\}$ is a loop that determines an element $\phi$ in the centralizer $\zeta(\gamma)$ of $\gamma$ in $\pi_{1} M$. This element $\phi$ is well-defined up to multiplication by powers of $\gamma$ and hence determines a well-defined element in the double coset space $\langle\gamma\rangle \backslash \pi_{1} M /\langle\gamma\rangle$.

In this setting we will speak of "the element $\phi \in \zeta(\gamma)$ determined by a latitude" since such an element represents a well-defined double coset.

The notation $H_{\phi}$ will sometimes be used to indicate that a singular concordance has a latitude whose projection represents $\phi$.

\subsection{Latitudes of singular self-concordances}

A singular self-concordance $K$ of a knot $k$ with whisker $w$ has a preferred latitude which uses $w$ at both ends of $K$. A latitude of a singular self-concordance will always be assumed to be such a preferred latitude and when it is necessary to carefully specify the whisker being used we will use the notation

$$
\phi=\operatorname{lat}[K](w)
$$

for the element $\phi \in \zeta(\gamma)$ determined by $K$ and $w$.

\subsection{Self-intersection numbers of singular concordances}

The self-intersection number $\mu(H)$ of a singular concordance $H$ of $k \in \mathcal{K}_{\gamma}(M)$ takes values in $\tilde{\Lambda}_{\gamma}$ and when necessary we use the notation $\mu(H)(w)$ to indicate that $\mu(H)$ is computed using a specific whisker $w$ for $k$. By our convention that $[k]=\gamma \in \pi_{1} M$, any two choices of whisker for $k$ differ by an element $\alpha \in \zeta(\gamma)$, that is, $\alpha=\left[w^{\prime}-w\right]$ is determined by the oriented loop $w^{\prime}-w$ which goes to $k$ along $w^{\prime}$ and back to the basepoint of $M$ along $w$. The effect of a whisker change on $\mu(H)$ is conjugation by $\alpha$ :

$$
\mu(H)\left(w^{\prime}\right)=\alpha(\mu(H)(w)) \alpha^{-1} .
$$




\subsection{Additivity and composition}

If $H$ is a singular concordance from $k$ to $k^{\prime}$ and $H^{\prime}$ is a singular concordance from $k^{\prime}$ to $j$ then we write $H+H^{\prime}$ for the singular concordance from $k$ to $j$ that is the composition of $H$ followed by $H^{\prime}$ (with $I$ reparametrized appropriately). If $w$ and $w^{\prime}$ are the whiskers for $k$ and $k^{\prime}$ we have

$$
\mu\left(H+H^{\prime}\right)=\mu(H)+\phi \mu\left(H^{\prime}\right) \phi^{-1}
$$

where $\phi$ is determined by a latitude of $H$ using the whiskers $w$ and $w^{\prime}$ (recall our convention that basepoints are taken in $M \times\{0\})$. We will be particularly concerned with the case where $k=k^{\prime}$ and $w=w^{\prime}$ so that $H$ is a singular self-concordance and $\phi=\operatorname{lat}[H](w)$.

Note that if a latitude of $H^{\prime}$ determines $\phi^{\prime}$ then $H+H^{\prime}$ has a latitude determining $\phi \phi^{\prime}$.

If $H$ is a singular concordance from $k$ to $j$ then we write $-H$ for the "inverse" singular concordance from $j$ to $k$ that inverts the $I$ parameter of $S^{1} \times I$ (which has the effect of changing all the signs of the singularities of $H$ ). For instance, if $H$ is the trace of a homotopy of a knot then $-H$ is the trace of the homotopy "run backwards." If a latitude of $H$ determines $\phi$, then $-H$ has a latitude determining $\phi^{-1}$ and

$$
\mu(-H)=-\phi^{-1} \mu(H) \phi .
$$

\subsection{The indeterminacy subgroup $\Phi(k)$}

Definition 4.7.1 For any knot $k \in \mathcal{K}_{\gamma}(M)$, define

$$
\Phi(k) \leq \tilde{\Lambda}_{\gamma} \rtimes \zeta(\gamma)
$$

to be the subgroup of the semi-direct product of $\tilde{\Lambda}_{\gamma}$ and $\zeta(\gamma)$ (with respect to the conjugation action of $\zeta(\gamma)$ on $\left.\tilde{\Lambda}_{\gamma}\right)$ generated by the elements

$$
(\mu(K)(w), \operatorname{lat}[K](w)) \in \tilde{\Lambda}_{\gamma} \rtimes \zeta(\gamma)
$$

as $K$ ranges over all singular self-concordances of $k$ in $M$ and $w$ ranges over all whiskers identifying $[k]=\gamma \in \pi_{1} M$.

\subsubsection{The action of $\Phi(k)$ on $\tilde{\Lambda}_{\gamma}$}

The group $\tilde{\Lambda}_{\gamma} \rtimes \zeta(\gamma)$ acts on $\tilde{\Lambda}_{\gamma}$ by

$$
(z, \phi): y \mapsto z+\phi y \phi^{-1}
$$


for $(z, \phi) \in \tilde{\Lambda}_{\gamma} \rtimes \zeta(\gamma)$ and $y \in \tilde{\Lambda}_{\gamma}$. Denote the equivalence classes under the restriction of this action to the subgroup $\Phi(k)$ by

$$
\tilde{\Lambda}_{\gamma} / \Phi(k) \text {. }
$$

The above action corresponds to the effect that pre-composing by a singular selfconcordance $K$ has on the self-intersection number of a singular concordance $H$ (4.6) provided that $\mu(K)$ and $\mu(H)$ are both computed using the same whisker.

\subsubsection{The action of $\zeta(\gamma)$ on $\tilde{\Lambda}_{\gamma} / \Phi(k)$}

The effect that changing the whisker for $k$ has on a generator of $\Phi(k)$ is described by the diagonal conjugation action of $\zeta(\gamma)$ on $\tilde{\Lambda}_{\gamma} \rtimes \zeta(\gamma)$ :

$$
\alpha:(z, \phi) \mapsto\left(\alpha z \alpha^{-1}, \alpha \phi \alpha^{-1}\right)
$$

for all $\alpha \in \zeta(\gamma)$.

We have a well-defined conjugation action of $\zeta(\gamma)$ on $\tilde{\Lambda}_{\gamma} / \Phi(k)$ since if

$$
x=z+\phi y \phi^{-1} \in \tilde{\Lambda}_{\gamma}
$$

we have

$$
\alpha x \alpha^{-1}=\alpha z \alpha^{-1}+\left(\alpha \phi \alpha^{-1}\right)\left(\alpha y \alpha^{-1}\right)\left(\alpha \phi^{-1} \alpha^{-1}\right) \in \tilde{\Lambda}_{\gamma} .
$$

We denote by

$$
\tilde{\Lambda}_{\gamma} /(\Phi(k), \zeta(\gamma))
$$

the orbit space of $\tilde{\Lambda}_{\gamma} / \Phi(k)$ under conjugation by $\zeta(\gamma)$.

Remark 4.7.2 In order to see the difficulties in putting a group structure on $\tilde{\Lambda}_{\gamma} /(\Phi(k), \zeta(\gamma))$, note that if $(\mu(K), \phi) \in \Phi(k)$ and $\phi \mu(K) \phi^{-1} \neq \mu(K) \in \tilde{\Lambda}_{\gamma}$, then $\mu(K)$ and $\mu(K)+\phi \mu(K) \phi^{-1}$ are each in the orbit of $0 \in \tilde{\Lambda}_{\gamma} /(\Phi(k), \zeta(\gamma))$ but $\mu(K)+\mu(K)=2 \mu(K)$ may not be in the orbit of 0 .

\subsection{Relative self-linking numbers}

We can now define the algebraic relative self-linking numbers $\mu_{k}(j)$; the adjective "algebraic" will usually be omitted in the interest of brevity.

Definition 4.8.1 Fix any knot $k$ in $\mathcal{K}_{\gamma}(M)$. For all $j \in \mathcal{K}_{\gamma}(M)$, define $\mu_{k}(j)$ the relative self-linking number of $j$ with respect to $k$ by

$$
\mu_{k}(j):=\mu(H) \in \tilde{\Lambda}_{\gamma} /(\Phi(k), \zeta(\gamma))
$$

where $H$ is any singular concordance from $k$ to $j$. 


\subsection{Proof of Theorem 2}

After unravelling equivalence relations, we have that $\mu_{k}(j)=\mu_{k}\left(j^{\prime}\right)$ if and only if for any singular concordances $H$ and $H^{\prime}$ from $k$ to $j$ and $j^{\prime}$, respectively, there exist an element $(z, \phi) \in \Phi(k)$ and an element $\alpha \in \zeta(\gamma)$ such that

$$
\alpha \mu(H) \alpha^{-1}=z+\phi \mu\left(H^{\prime}\right) \phi^{-1} \in \tilde{\Lambda}_{\gamma} .
$$

Here $\mu(H)$ and $\mu\left(H^{\prime}\right)$ are computed using whiskers $w$ and $w^{\prime}$, respectively, on $k$ such that $[k]=\gamma$.

Independence of choice of singular concordance If $H$ and $H^{\prime}$ are any two singular concordances from $k$ to $j$, then the composition $K:=H-H^{\prime}$ is a singular self-concordance of $k$ (see Figure 5). Using the whisker $w^{\prime}$ on $k$ as a whisker for $K$, we have

$$
\mu(K)=\alpha \mu(H) \alpha^{-1}-\phi \mu\left(H^{\prime}\right) \phi^{-1} \in \tilde{\Lambda}_{\gamma}
$$

where $\alpha \in \zeta(\gamma)$ is determined by the difference of the whiskers $w$ and $w^{\prime}$ and $\phi \in \zeta(\gamma)$ is determined by a latitude of $K$ (using the whisker $w^{\prime}$ ). Thus, the relative self-linking number $\mu_{k}(j)$ does not depend on the choice of singular concordance from $k$ to $j$ since the equation

$$
\alpha \mu(H) \alpha^{-1}=\mu(K)+\phi \mu\left(H^{\prime}\right) \phi^{-1}
$$

in $\tilde{\Lambda}_{\gamma}$ shows that

$$
\mu(H)=\mu\left(H^{\prime}\right) \in \tilde{\Lambda}_{\gamma} /(\Phi(k), \zeta(\gamma)) .
$$

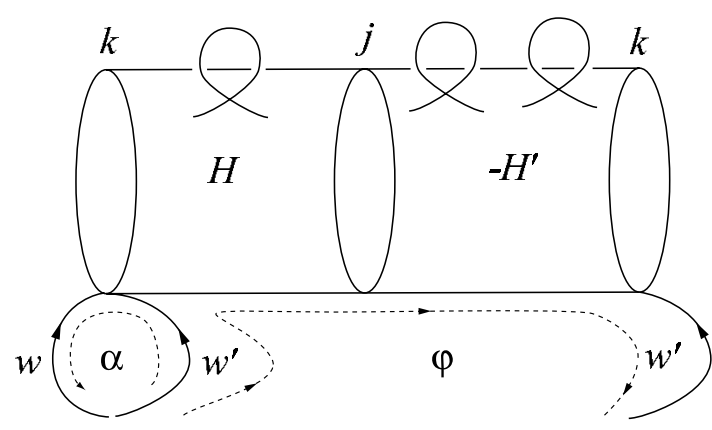

Figure 5

Algebraic 63 Geometric Topology, Volume 3 (2003) 
Concordance invariance First we show that concordant knots have the same indeterminacy subgroup. Let $C$ be any concordance from $k^{\prime}$ to $k$. Then for any singular self-concordance $K$ of $k$, the composition $K^{\prime}:=C+K-C$ is a singular self-concordance of $k^{\prime}$ and, since $\mu(C)=0$, we have

$$
\mu\left(K^{\prime}\right)=\mu(C+K-C)=\alpha \mu(K) \alpha^{-1}
$$

where $\alpha \in \zeta(\gamma)$ is determined by a latitude of $C$ which depends on the whiskers for $k$ and $k^{\prime}$. By choosing an appropriate whisker for $k^{\prime}$ it can be arranged that $\alpha=1$, which means that

$$
\left(\mu\left(K^{\prime}\right), \phi^{\prime}\right)=(\mu(K), \phi) \in \tilde{\Lambda}_{\gamma} \rtimes \zeta(\gamma)
$$

where $\phi$ and $\phi^{\prime}$ are determined by latitudes of $K$ and $K^{\prime}$ respectively. Thus, $\Phi(k) \leq \Phi\left(k^{\prime}\right)$ and by a symmetric argument $\Phi(k)=\Phi\left(k^{\prime}\right)$.

Now we also vary $j$ by a concordance. If $C^{\prime}$ is any concordance from $j$ to $j^{\prime}$, then for any singular concordance $H$ from $k$ to $j$ the composition $H^{\prime}:=$ $C+H+C^{\prime}$ is a singular concordance from $k^{\prime}$ to $j^{\prime}$ and, since $C$ and $C^{\prime}$ have no singularities, we have

$$
\mu_{k^{\prime}}\left(j^{\prime}\right)=\mu\left(H^{\prime}\right)=\alpha \mu(H) \alpha^{-1}=\mu_{k}(j) \in \tilde{\Lambda}_{\gamma} /(\Phi(k), \zeta(\gamma))
$$

where $\alpha \in \zeta(\gamma)$ is determined by a latitude of the concordance $C$ from $k^{\prime}$ to $k$. Thus, the induced map in the statement of Theorem 2 is well-defined.

The induced map is onto To see that $\mathcal{C}_{\gamma}(M)$ is mapped onto the target space, just apply the same construction as in the proof Theorem 5 (see 3.2): push out small arcs of $k$ around loops and create \pm -clasps with $k$.

\section{$5 \quad$ The indeterminacy sub-group $\Phi(k)$}

In this section we take up the question of computability of relative self-linking numbers. This computability depends to large extent on describing the indeterminacy subgroup $\Phi(k)$. The main goal of the section is Proposition 5.4.1 which describes a set of generators for $\Phi(k)$. The indeterminacies come from two kinds of self-homotopies: Spherical self-homotopies (5.2) contribute indeterminacies that can be measured by an intersection pairing between spheres and knots (5.1) and indeterminacies coming from toriodal self-homotopies (5.3) correspond to essential maps of tori and can be checked on a generating set for the centralizer $\zeta(\gamma)$ of $\gamma=[k]$. Lemma 5.2.1 and its corollary present the key 
observation that the spherical self-homotopies are precisely those with trivial latitudes.

In fact, the generating set of $\Phi(k)$ often simplifies significantly in practice, as described in the paragraphs following the statement of Proposition 5.4.1 and as illustrated in the examples of Section 8.

\subsection{Intersecting knots and spheres}

Let $k$ be a knot in $\mathcal{K}_{\gamma}(M)$ and $S$ be an immersed 2-sphere in $M$ equipped with whiskers so that $[k]=\gamma \in \pi_{1} M$ and $[S]=\sigma \in \pi_{2} M$. To each intersection point $p \in k \cap S$, associate an element $g_{p} \in \pi_{1} M$ from a loop going from the basepoint of $M$ along the whisker on $S$, along $S$ to $p$ (without changing sheets at any singularities of $S$ ), along $k$, then back along the whisker on $k$ to the basepoint of $M$. The sum of the $g_{p}$, taken in $\Lambda_{\gamma}$, only depends on the homotopy classes of $k$ and $S$.

Definition 5.1.1 For $k \in \mathcal{K}_{\gamma}(M)$ and $\sigma \in \pi_{2} M$, define

$$
\lambda(\sigma, k):=\sum(\operatorname{sign} p) \cdot g_{p} \in \Lambda_{\gamma}
$$

where the sum is over all intersection points $p$ between $k$ and any immersed 2 -sphere representing $\sigma$.

Changing the whisker identifying $[k]=\gamma$ changes $\lambda(\sigma, k)$ by right multiplication by a difference of whiskers in $\zeta(\gamma)$.

We will use the following linearity property of $\lambda(\sigma, k)$ :

$$
\lambda\left(g_{1} \cdot \sigma_{1}+g_{2} \cdot \sigma_{2}, k\right)=g_{1} \lambda\left(\sigma_{1}, k\right)+g_{2} \lambda\left(\sigma_{2}, k\right)
$$

for all $g_{1}, g_{2} \in \pi_{1} M$ and $\sigma_{1}, \sigma_{2} \in \pi_{2} M$.

\subsection{Spherical self-homotopies}

Pushing a local strand of a knot $k \in \mathcal{K}_{\gamma}(M)$ around a loop representing $g \in$ $\pi_{1} M$, then across an embedded 2-sphere $S$ as indicated in Figure 6, creates a spherical self-homotopy of $k$. Denoting by $K$ the trace in $M \times I$ of such a spherical self-homotopy, we have

$$
\mu(K)=\widetilde{\lambda}(g \cdot \sigma, k) \in \tilde{\Lambda}_{\gamma}
$$




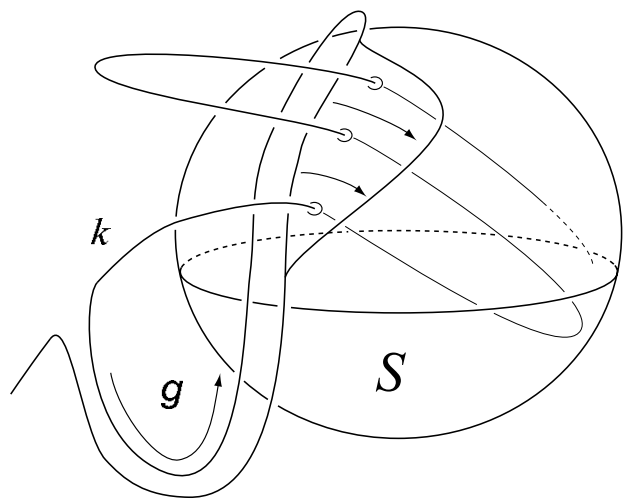

Figure 6

where $S$ (appropriately based) represents $\sigma \in \pi_{2} M$ and $\widetilde{\lambda}(\sigma, k)$ is $\lambda(\sigma, k)$ taken in the quotient $\tilde{\Lambda}_{\gamma}$ of $\Lambda_{\gamma}$. Note that $K$ has a latitude which determines the trivial element of $\pi_{1} M$. The next lemma and its corollary give a converse to this observation.

Lemma 5.2.1 If a singular self-concordance $K$ of a knot $k \subset M$ has a latitude that represents the trivial element $1 \in \pi_{1} M$, then $K$ is homotopic (rel $\partial$ ) to the connected sum of an embedded 2-sphere and the embedded annulus $A=k \times I$ in $M \times I$.

Proof The projection $T$ of $K$ in $M$ is a map of a torus into $M$. This map $T$ extends to a map (we will still call $T$ ) of a torus with a 2-disk $D$ attached along the homotopically trivial loop which is the projection of the latitude. The obstruction to further extending $T$ to a map of a solid torus is an immersed 2 -sphere $S$ in $M$ which is the result of using (the image of) $D$ to surger $T$.

If $S$ bounds an immersed 3-ball in $M$, then $T$ extends to a map of a solid torus $S^{1} \times D^{2}$ with $k$ corresponding to the $S^{1}$ factor. (This solid torus is the union of the 3 -ball and $D \times I$ joined along $D \times\{0,1\}$.) In this case, by collapsing along the $D^{2}$ factor (as indicated in Figure $7(\mathrm{a})$ ) we get a homotopy from $T$ to $k$ which lifts to a homotopy (rel $\partial$ ) from $K$ to $A$.

If $S$ does not bound a map of a 3-ball, then $T$ deformation retracts onto the connected sum of $S$ with a small band on $k$ (Figure 7(b)). In this case the retraction lifts to a homotopy (rel $\partial$ ) from $K$ to the connected sum $A^{\prime}$ of $A$ with a 2 -sphere $S^{\prime}$ that is a lift of $S$ (see Figure 8). By Lemma 3.2.1 we can assume that $S^{\prime}$ is embedded. 


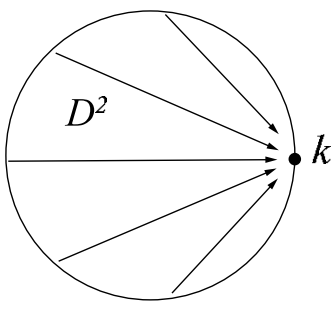

(a)

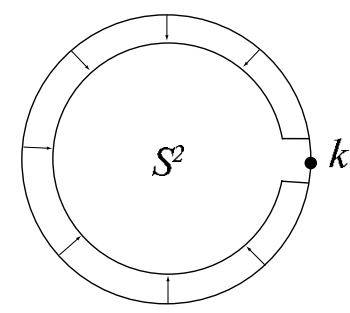

(b)

Figure 7

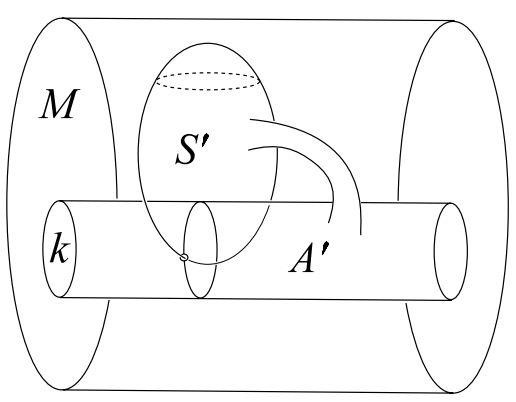

Figure 8

Corollary 5.2.2 If $K$ is a singular self-concordance of any knot $k$ in $\mathcal{K}_{\gamma}(M)$ and $K$ has a latitude which determines the trivial element of $\pi_{1} M$, then $\mu(K)=$ $\widetilde{\lambda}(\sigma, k)$ for some $\sigma \in \pi_{2} M$.

Proof By Lemma 5.2.1 and the homotopy invariance of $\mu$, we have that $\mu(K)=\mu\left(A^{\prime}\right)$, where $A^{\prime}$ is the connected sum of an embedded 2-sphere $S^{\prime}$ and the embedded product annulus $A=k \times I$ in $M \times I$. The embedded 2-sphere $S^{\prime}$ consists of parallel copies of embedded generating 2-spheres in different copies of $M$ cross a point in $M \times I$ tubed together as described in the proof of Lemma 3.2.1. Thus, the only singularities of the annulus $A^{\prime}$ come from intersections between $k$ and the embedded 2-spheres in $M$. The corollary follows by additivity (5.1) with $\sigma=\left[S^{\prime}\right]$.

\subsection{Toroidal self-homotopies}

For each non-trivial element $\phi \in \zeta(\gamma)$, the equation $\gamma \phi \gamma^{-1} \phi^{-1}=1 \in \pi_{1} M$ corresponds (after a choice of a null-homotopy) to a map of a torus into $M$. For $k \in \mathcal{K}_{\gamma}(M)$, such a map parametrizes a self-homotopy $K_{\phi}$ which has a latitude 
representing $\phi$. The next lemma states that the self-intersection number of the trace of $K_{\phi}$ is unique up to spherical intersections. Such toroidal selfhomotopies will appear in Proposition 5.4.1 below.

Lemma 5.3.1 For $k \in \mathcal{K}_{\gamma}(M)$, if $K_{\phi}$ and $K_{\phi}^{\prime}$ are singular self-concordances of $k$ with

$$
\operatorname{lat}[K](w)=\operatorname{lat}\left[K^{\prime}\right](w) \phi \in \zeta(\gamma)
$$

for some whisker $w$ on $k$ then

$$
\mu\left(K_{\phi}\right)(w)-\mu\left(K_{\phi}^{\prime}\right)(w)=\lambda(\sigma, k)
$$

for some $\sigma \in \pi_{2} M$.

Proof Apply Corollary 5.2.2 to $K_{\phi}-K_{\phi}^{\prime}$.

\section{$5.4 \quad$ A generating set for $\Phi(k)$}

Proposition 5.4.1 Given $k \in \mathcal{K}_{\gamma}(M)$, let $\left\{\phi_{q}\right\}$ be any generating set for the centralizer $\zeta(\gamma)$ and let $\left\{K_{q}\right\}$ be any set of traces of self-homotopies of $k$ such that, for a fixed whisker $w_{0}$ on $k$, lat $\left[K_{q}\right]\left(w_{0}\right)=\phi_{q}$ for each $q$. Let $\left\{\sigma_{s}\right\}$ be any generating set for $\pi_{2} M$ as a module over $\pi_{1} M$. Denote by $\mathcal{S}_{0}$ the set

$$
\mathcal{S}_{0}:=\left\{\left(\mu\left(K_{q}\right)\left(w_{0}\right), \phi_{q}\right),\left(g \widetilde{\lambda}\left(\sigma_{s}, k\right)\left(w_{0}\right), 1\right)\right\}
$$

where $g$ ranges over $\pi_{1} M$.

Then $\Phi(k)$ is generated by

$$
\bigcup_{\alpha \in \zeta(\gamma)} \alpha \mathcal{S}_{0} \alpha^{-1}
$$

where the conjugation $\alpha \mathcal{S}_{0} \alpha^{-1}$ of $\mathcal{S}_{0}$ by $\alpha$ denotes conjugation of each element of $\mathcal{S}_{0}$ by $\alpha$.

The generating set described in Proposition 5.4.1 will often simplify significantly in practice. Usually $\mathcal{S}_{0}$ will contain either non-trivial spherical indeterminacies or non-trivial toroidal indeterminacies but not both. Also, the conjugation action by $\zeta(\gamma)$ is often trivial; for instance if $\gamma$ is primitive and $\zeta(\gamma)$ is cyclic.

If $M$ is irreducible, then $\zeta(\gamma)$ is finitely generated [14] and there are no spherical indeterminacies so $\mathcal{S}_{0}$ is a finite set. However, if $M$ is not irreducible and $k$ has non-trivial intersections with a $2-$ sphere, then $\Phi(k)$ is, in general, infinitely presented as will be illustrated in Section 8 .

Before giving a proof of Proposition 5.4.1, we give an example which will also serve to illustrate constructions used later in the proof of Theorem 4. 
Example 5.4.2 Consider the knot $k \in \mathcal{K}_{x y z}(M)$ in Figure 1 of the introduction, where $M$ is the product of a thrice punctured 2-disk with the circle. In this case $M$ is irreducible, so there are no generators of $\Phi(k)$ coming from spherical intersections and $\zeta(x y z)$ is isomorphic to $\mathbb{Z} \oplus \mathbb{Z}$ generated by $x y z$ and $t ; \Phi(k)$ can be computed by constructing just two self-homotopies of $k$ with latitudes representing $x y z$ and $t$ respectively. Two such self-isotopies of $k$ can easily be constructed, one by isotoping $k$ along itself longitudinally and the other by isotoping $k$ around the circle fiber of $M$. Since the trace of a self-isotopy is an embedded annulus, and hence has vanishing self-intersection number in $\widetilde{\Lambda}_{\gamma}$, the set $\mathcal{S}_{0}$ of Proposition 5.4.1 contains only the two elements $(0, x z y)$ and $(0, t)$ which shows that the action $\Phi(k)$ reduces to conjugation by $\zeta(x y z)$. Since $t$ is central in $\pi_{1} M$ and $x y z$ is primitive, it follows that the conjugation action of $\zeta(x y z)$ on $\widetilde{\Lambda}_{x y z}$ is trivial, justifying the statement that the target space for relative self-linking numbers with respect to $k$ is $\widetilde{\Lambda}_{x y z}$ itself.

\subsection{Proof of Proposition 5.4.1}

Let $(\mu(K)(w), \phi)$ be any generator of $\Phi(k) \leq \tilde{\Lambda}_{\gamma} \rtimes \zeta(\gamma)$; that is, $K$ is a singular self-concordance of $k$ such that $\operatorname{lat}[K](w)=\phi \in \zeta(\gamma)$ for some whisker $w$.

First consider the case when $w=w_{0}$ : Since the $\phi_{q}$ generate $\zeta(\gamma)$, there exists a finite sequence $K_{q_{i}}$ of the $\left\{K_{q}\right\}$ such that

$$
K^{\prime}:=K+\sum_{i} K_{q_{i}}
$$

is a singular self-concordance of $k$ with lat $\left[K^{\prime}\right]\left(w_{0}\right)=1 \in \pi_{1} M$.

By Corollary 5.2.2,

$$
\mu\left(K^{\prime}\right)\left(w_{0}\right)=\tilde{\lambda}(\sigma, k)\left(w_{0}\right)=\sum_{s} \tilde{\lambda}\left(g_{s} \cdot \sigma_{s}, k\right)\left(w_{0}\right)
$$

for some $\sigma \in \pi_{2} M$.

We have the following equation in $\tilde{\Lambda}_{\gamma} \rtimes \zeta(\gamma)$ :

$$
\left(\mu(K)\left(w_{0}\right), \phi\right) \prod_{i}\left(\mu\left(K_{q_{i}}\right)\left(w_{0}\right), \phi_{q_{i}}\right)=\prod_{s}\left(g_{s} \widetilde{\lambda}\left(\sigma_{s}, k\right)\left(w_{0}\right), 1\right)
$$

which can be solved for $\left(\mu(K)\left(w_{0}\right), \phi\right)$ in terms of elements (and their inverses) from $\mathcal{S}_{0}$.

For the general case where $w$ and $w_{0}$ differ by $\alpha \in \zeta(\gamma)$, the analogous construction expresses $(\mu(K)(w), \phi)$ in terms of elements from $\alpha \mathcal{S}_{0} \alpha^{-1}$. 


\section{Spherical knots and the proof of Theorem 3}

The proof of Theorem 3 is given in this section after first defining spherical knots and stating a lemma which isolates the properties of spherical knots to be used in the proof. The proof of the lemma is deferred until the end of the section. Here is the key point: For a spherical knot, every element of the centralizer can be realized as a latitude of a self-homotopy with vanishing selfintersection number; this means that the conjugation effect, which in general obstructs additivity of $\mu$ under composition of singular concordances (4.6), can always be "cancelled out" by inserting these self-homotopies accordingly.

\subsection{Spherical knots}

Definition 6.1.1 A knot $k_{0} \in \mathcal{K}_{\gamma}$ is spherical if there exists a whisker $w_{0}$ on $k_{0}$ such that for any $\phi \in \zeta(\gamma)$ there exists a singular self-concordance $K_{\phi}^{0}$ of $k_{0}$ with lat $\left[K_{\phi}^{0}\right]\left(w_{0}\right)=\phi$ and $\mu\left(K_{\phi}^{0}\right)\left(w_{0}\right)=0 \in \tilde{\Lambda}_{\gamma}$.

By Proposition 5.4.1, " $k_{0}$ is spherical" means that " $\Phi\left(k_{0}\right)$ contains only spherical indeterminacies," that is, indeterminacies due to intersections between $k_{0}$ and $2-$ spheres in $M$.

Example 6.1.2 The knot $k$ in Figure 1 of the introduction is spherical by the discussion in Example 5.4.2 and any knot in $S^{1} \times S^{2}$ is spherical as will be discussed in 8.1.

Remark For any two spherical knots $k_{0}$ and $k_{0}^{\prime}$ in $\mathcal{K}_{\gamma}$, we have $\Phi\left(k_{0}\right)=\Phi\left(k_{0}^{\prime}\right)$ since intersections between knots and spheres only depend on the homotopy classes.

The next lemma shows that, for spherical knots, all elements of the indeterminacy sub-group can be realized by actual singular self-concordances. (This is not true in general for non-spherical knots! See remarks 4.7.2 and 6.3.1.)

Lemma 6.1.3 If $k \in \mathcal{K}_{\gamma}(M)$ is spherical, then

(i) for any $\alpha \in \zeta(\gamma)$ and any whisker $w$ on $k$, there exists a singular selfconcordance $K_{\alpha}^{0}$ of $k$ such that $\operatorname{lat}\left[K_{\alpha}^{0}\right](w)=\alpha$ and

$$
\mu\left(K_{\alpha}^{0}\right)(w)=0 \in \tilde{\Lambda}_{\gamma}
$$


(ii) for every $(z, \phi) \in \Phi(k)$ and every whisker $w$ on $k$, there exists a singular self-concordance $K_{\phi}^{z}$ of $k$ such that $\operatorname{lat}\left[K_{\phi}^{z}\right](w)=\phi$ and

$$
\mu\left(K_{\phi}^{z}\right)(w)=z \in \tilde{\Lambda}_{\gamma} .
$$

The proof of this lemma is deferred until the end of this section.

\subsection{Proof of Theorem 3}

Proof of (i) $\Rightarrow$ (ii) We will construct a singular concordance $H^{0}$ between $j$ and $j^{\prime}$ such that $\mu\left(H^{0}\right)=0 \in \tilde{\Lambda}_{\gamma}$ which by Proposition 4.1.2 implies (ii).

The equality of $\mu_{k}(j)$ and $\mu_{k}\left(j^{\prime}\right)$ means that for any singular concordances $H$ and $H^{\prime}$ from $k$ to $j$ and $j^{\prime}$, respectively, there exists $\alpha \in \zeta(\gamma)$ and $(z, \phi) \in \Phi(k)$ such that

$$
\alpha \mu(H) \alpha^{-1}=(z, \phi) \cdot \mu\left(H^{\prime}\right) \in \tilde{\Lambda}_{\gamma} .
$$

Since this holds for any such $H$ and $H^{\prime}$, we may assume that $H$ and $H^{\prime}$ have the same whisker $w$ on $k$ so that

$$
\alpha(\mu(H)(w)) \alpha^{-1}=z+\phi\left(\mu\left(H^{\prime}\right)(w) \phi^{-1} \in \tilde{\Lambda}_{\gamma} .\right.
$$

By Lemma 6.1.3, there exist singular self-concordances $K_{\alpha}^{0}$ and $K_{\phi}^{z}$ of $k$ such that $\mu\left(K_{\alpha}^{0}\right)(w)=0$ and $\mu\left(K_{\phi}^{z}\right)(w)=z$ in $\tilde{\Lambda}_{\gamma}$ with $\operatorname{lat}\left[K_{\alpha}^{0}\right](w)=\alpha$ and $\operatorname{lat}\left[K_{\phi}^{z}\right](w)=\phi$.

The composition $H^{0}:=-H-K_{\alpha}^{0}+K_{\phi}^{z}+H^{\prime}$ is a singular concordance from $j$ to $j^{\prime}$ (see Figure 9). Using the whisker $w$ in the intersection $\left(-K_{\alpha}^{0} \cap K_{\phi}^{z}\right) \subset H^{0}$ as a whisker for $H^{0}$, we have

$$
\begin{aligned}
\mu\left(H^{0}\right)(w) & =\mu\left(-H-K_{\alpha}^{0}+K_{\phi}^{z}+H^{\prime}\right)(w) \\
& =-\alpha(\mu(H)(w)) \alpha^{-1}+0+z+\phi\left(\mu\left(H^{\prime}\right)(w)\right) \phi^{-1} \\
& =0 \in \tilde{\Lambda}_{\gamma}
\end{aligned}
$$

which, by Proposition 4.1.2, completes the proof that (i) implies (ii).

Proof of (ii) $\Rightarrow$ (i) Let $J$ be a singular concordance from $j$ to $j^{\prime}$ with all singularities of $J$ paired by Whitney disks. By Proposition 4.1.2, $\mu(J)=0 \in$ $\tilde{\Lambda}_{\gamma}$. If $H$ is any singular concordance from $k$ to $j$, the composition $H+J$ is a singular concordance from $k$ to $j^{\prime}$ and we have

$$
\mu_{k}(j)=\mu(H)=\mu(H+J)=\mu_{k}\left(j^{\prime}\right)
$$

since all singularities of $J$ come in cancelling pairs. 


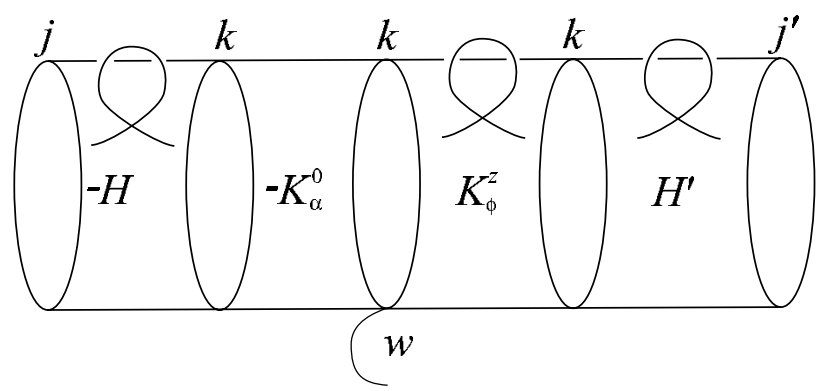

Figure 9

\subsection{Proof of Lemma 6.1.3}

Proof of property (i) of Lemma 6.1.3 Property (i) follows from the fact that changing whiskers on $k$ corresponds to conjugation by $\zeta(\gamma)$ which induces isomorphisms of $\tilde{\Lambda}_{\gamma}$ and $\zeta(\gamma)$.

Specifically, given $\alpha \in \zeta(\gamma)$ and any whisker $w$ on $k$, if $\psi \in \zeta(\gamma)$ is determined by the difference $\left[w-w_{0}\right]$ of $w$ and $w_{0}$ (where $w_{0}$ is the whisker of Definition 6.1.1), then, for $K_{\psi^{-1} \alpha \psi}^{0}$ with lat $\left[K_{\psi^{-1} \alpha \psi}^{0}\right]\left(w_{0}\right)=\psi^{-1} \alpha \psi$ and $\mu\left(K_{\psi^{-1} \alpha \psi}^{0}\right)\left(w_{0}\right)=0 \in \tilde{\Lambda}_{\gamma}$ as guaranteed by Definition 6.1.1, we have

$$
\operatorname{lat}\left[K_{\psi^{-1} \alpha \psi}^{0}\right](w)=\psi \operatorname{lat}\left[K_{\psi^{-1} \alpha \psi}^{0}\right]\left(w_{0}\right) \psi^{-1}=\alpha
$$

and

$$
\mu\left(K_{\psi^{-1} \alpha \psi}^{0}\right)(w)=\psi \mu\left(K_{\psi^{-1} \alpha \psi}^{0}\right)\left(w_{0}\right) \psi^{-1}=0 \in \tilde{\Lambda}_{\gamma}
$$

Proof of property (ii) of Lemma 6.1.3 Let $K_{\phi}^{z}$ be a singular self-concordance of $k$ with

$$
(z, \phi)=\left(\mu\left(K_{\phi}^{z}\right)\left(w_{0}\right), \operatorname{lat}\left[K_{\phi}^{z}\right]\left(w_{0}\right)\right)
$$

for some whisker $w_{0}$ on $k$ and let $w$ be any other whisker on $k$ with $\psi=$ $\left[w-w_{0}\right] \in \zeta(\gamma)$. By property (i) of Lemma 6.1.3, there exists a singular selfconcordance $K_{\psi^{-1}}^{0}$ of $k$ such that lat $\left[K_{\psi^{-1}}^{0}\right](w)=\psi^{-1}$ and $\mu\left(K_{\psi^{-1}}^{0}\right)(w)=0 \in$ $\tilde{\Lambda}_{\gamma}$. The composition $K^{\prime}:=K_{\psi^{-1}}^{0}+K_{\phi}^{z}-K_{\psi^{-1}}^{0}$ satisfies

$$
\operatorname{lat}\left[K^{\prime}\right](w)=\psi^{-1}\left(\operatorname{lat}\left[K_{\phi}^{z}\right](w)\right) \psi=\psi^{-1} \psi\left(\operatorname{lat}\left[K_{\phi}^{z}\right]\left(w_{0}\right)\right) \psi^{-1} \psi=\phi
$$

and

$$
\mu\left(K^{\prime}\right)(w)=\psi^{-1}\left(\mu\left(K_{\phi}^{z}\right)(w)\right) \psi=\psi^{-1} \psi\left(\mu\left(K_{\phi}^{z}\right)\left(w_{0}\right)\right) \psi^{-1} \psi=z .
$$


Thus, to prove property (ii) of Lemma 6.1.3 it suffices to show that it holds for a fixed whisker $w_{0}$, that is, it suffices to show that for any $(z, \phi) \in \Phi(k)$ there exists $K_{\phi}^{z}$ satisfying the above equation $(*)$ for $w_{0}$.

Since $k$ is spherical, by Proposition 5.4.1 $\Phi(k)$ is generated by the set

$$
\mathcal{S}=\left\{\left(0, \phi_{q}\right),\left(\alpha g \widetilde{\lambda}\left(\sigma_{s}, k\right)\left(w_{0}\right) \alpha^{-1}, 1\right)\right\}
$$

for some whisker $w_{0}$ on $k$, where $\alpha$ ranges over $\zeta(\gamma)$, the $\phi_{q}$ generate $\zeta(\gamma)$, the $\sigma_{s}$ generate $\pi_{2} M$ as a module over $\pi_{1} M$, and $g$ ranges over $\pi_{1} M$.

The cases $(z, \phi)=\left(0, \phi_{q}\right)$ (or more generally $z=(0, \phi)$ for any $\phi \in \zeta(\gamma)$ and any $w$ ) are covered by property (i) of Lemma 6.1 .3 (which is essentially built into Definition 6.1.1).

If $(z, \phi)=\left(g \widetilde{\lambda}\left(\sigma_{s}, k\right)\left(w_{0}\right), 1\right)$ then, as described in 5.2, there exists a spherical self-homotopy of $k$ whose trace $K_{g S_{s}}$ satisfies $\operatorname{lat}\left[K_{g S_{s}}\right]\left(w_{0}\right)=1$ and $\mu\left(K_{g S_{s}}\right)\left(w_{0}\right)=g \widetilde{\lambda}\left(\sigma_{s}, k\right)\left(w_{0}\right) \in \tilde{\Lambda}_{\gamma}$ where $g S_{s}$ is a 2 -sphere in $M$ representing $g \cdot \sigma_{s} \in \pi_{2} M$.

Conjugating by a singular self-concordance $K_{\alpha}^{0}$ of $k$ from property (i) yields a singular self-concordance $K_{\alpha}^{0}+K_{g S_{i}}-K_{\alpha}^{0}$ which satisfies $\left(^{*}\right)$ for $(z, \phi)=$ $\left(\alpha g \widetilde{\lambda}\left(\sigma_{s}, k\right)\left(w_{0}\right) \alpha^{-1}, 1\right)$.

We have found singular self-concordances satisfying $(*)$ for all elements of the generating set $\mathcal{S}$ for $\Phi(k)$. It only remains to observe that if $K$ and $K^{\prime}$ are any singular self-concordances of $k$ with lat $[K]\left(w_{0}\right)=\phi$ and lat $\left[K^{\prime}\right]\left(w_{0}\right)=\phi^{\prime}$, then $K+K^{\prime}$ satisfies $(*)$ for

$$
(z, \phi)=\left(\mu(K)\left(w_{0}\right), \phi\right)\left(\mu\left(K^{\prime}\right)\left(w_{0}\right), \phi^{\prime}\right)=\left(\mu(K)\left(w_{0}\right)+\phi\left(\mu\left(K^{\prime}\right)\left(w_{0}\right)\right) \phi^{-1}, \phi \phi^{\prime}\right) .
$$

Remark 6.3.1 It follows from Lemma 6.1.3, together with the observation that $(\mu(-K)(w)$, lat $[-K](w))$ is an inverse for $(\mu(K)(w)$, lat $[K](w))$, that if $k$ is spherical, then the subset

$$
\{(\mu(K)(w), \operatorname{lat}[K](w))\} \subset \tilde{\Lambda}_{\gamma} \rtimes \zeta(\gamma),
$$

as $K$ ranges over all singular self-concordances of $k$ in $M$ and $w$ ranges over all whiskers identifying $[k]=\gamma \in \pi_{1} M$, is actually equal to the subgroup $\Phi(k)$. The subtlety here is that if a knot is not spherical, then its indeterminacy subgroup can contain elements that are not realized by actual singular self-concordances of the knot. 


\section{The class $\mathcal{M}$ and proof of Theorem 4}

The proof of Theorem 4 is given in this section after the definition of the set $\mathcal{M}$. The subset of irreducible manifolds in $\mathcal{M}$ was denoted $\mathcal{N}$ in [19] and [20].

\subsection{The class $\mathcal{M}$}

Definition 7.1.1 Let $\mathcal{M}$ denote the set of all 3-manifolds $M$ such that

(i) $M$ does not contain any circle bundle over a non-orientable surface whose total space is orientable,

(ii) $M$ does not contain any of the Seifert manifolds $M\left(S^{2},(3,1),(3,1)\right.$, $(3,-2)), M\left(S^{2},(2,1),(4,-1),(4,-1)\right)$ or $M\left(S^{2},(2,1),(3,-1),(6,-1)\right)$.

Here "contain" means either as a submanifold or as a (punctured) prime factor and the notation $M\left(S^{2},(3,1),(3,1),(3,-2)\right)$, for instance, refers to the Seifert fibered manifold with orbit surface $S^{2}$ and three singular fibers having Seifert invariants $(3,1),(3,1)$ and $(3,-2)$. The excluded manifolds are exactly those which contain essential tori that contribute non-trivial indeterminacies to $\Phi(k)$ for all $k \in \mathcal{K}_{\gamma}(M)$ for certain $\gamma$ (see [19, 20, 24]).

\subsection{Proof of Theorem 4}

To show that a knot $k_{0}$ is spherical it is sufficient, by Proposition 5.4.1, to find self-homotopies of $k_{0}$ whose traces $K_{q}^{0}$ satisfy

$$
\left(\mu\left(K_{q}^{0}\right)\left(w_{0}\right), \operatorname{lat}\left[K_{q}^{0}\right]\left(w_{0}\right)\right)=\left(0, \phi_{q}\right) \in \tilde{\Lambda}_{\gamma} \rtimes \zeta(\gamma)
$$

for some whisker $w_{0}$ on $k_{0}$ where the $\phi_{q}$ generate $\zeta(\gamma)$. We will in fact be able to find self-isotopies of $k_{0}$ in all cases.

The proof breaks into different cases according to the structure of $\zeta(\gamma)$.

Null-homotopic knots For the case $\gamma=1$ take $k_{0}$ to be the unknot, that is, $k_{0}$ bounds an embedded 2-disk in $M$ (all such $k_{0}$ are clearly isotopic in any connected manifold). Now $\zeta(\gamma)=\pi_{1} M$ and $k_{0}$ can be isotoped into a neighborhood of a point and then isotoped around any loop which represents a generator of $\pi_{1} M$, completing the null-homotopic case. 
Remark If $k_{0}$ is the unknot, then the action of $\Phi\left(k_{0}\right)$ reduces to conjugation (since null-homotopic knots have trivial intersections with spheres) so relative self-linking numbers reduce to (absolute) self-linking numbers: $\mu_{k_{0}}(j)=\mu(j)$ for all $j \in \mathcal{K}_{1}(M)$.

From now on in the proof we will assume that $\gamma \neq 1$.

Cyclic centralizers and longitudinal self-isotopies Consider now the case where $\zeta(\gamma)$ is a cyclic subgroup of $\pi_{1}(M)$ generated by an element $\rho$ (possibly $\rho=\gamma$ ). Take $k_{0} \in \mathcal{K}_{\gamma}(M)$ to be contained in a tubular neighborhood $R$ of any knot representing $\rho$. An ambient self-isotopy of $R$ in $M$ that fixes $R$ set-wise but moves once around in the direction of $\rho$ induces a self-isotopy of $k_{0}$ whose trace $K_{\rho}^{0}$ has a latitude representing $\rho$. Such a self-isotopy will be referred to as a longitudinal self-isotopy. This completes the case where $\zeta(\gamma)$ is cyclic.

Remark 7.2.1 Note that in the case where $\zeta(\gamma)$ is cyclic there exists a spherical knot in $\mathcal{K}_{\gamma}(M)$ for any $M$.

So we may now assume that $\zeta(\gamma)$ and $\pi_{1}(M)$ are not cyclic groups.

The non-cyclic centralizer case In this setting, the classical 3-manifold structure theorems imply that $\zeta(\gamma)$ is carried by an embedded Seifert fibered submanifold of $M$ : By the Kneser/Milnor prime factorization theorem, $M$ has a unique connected sum decomposition into irreducible submanifolds and copies of $S^{1} \times S^{2}$ which give a decomposition of $\pi_{1} M$ as a free product. If $\gamma$ projected non-trivially to more than one free factor, then $\zeta(\gamma)$ would be cyclic (by Corollary 4.1.6 of [21]), so we may assume that $\gamma$ is carried by an irreducible factor $M_{\gamma} \subset M$. The irreducible submanifold $M_{\gamma}$ carries $\zeta(\gamma)$, and if $M_{\gamma}$ is Haken, then by the work of Jaco-Shalen and Johansson $\zeta(\gamma)$ is carried by a Seifert fibered submanifold of $M_{\gamma}$ (e.g. Theorem 4.1 of [16]). If $M_{\gamma}$ is not Haken, then by the theorems of Casson-Jungreis [1] and Gabai [8] either $\zeta(\gamma)$ is carried by a collar of a torus boundary component of $M_{\gamma}$ (which is Seifert fibered as $S^{1} \times I \times S^{1}$ ) or $M_{\gamma}$ is itself Seifert fibered.

Let $N \subset M_{\gamma} \subset M$ denote the embedded Seifert fibered sub-manifold that carries $\zeta(\gamma)$. The orbit surface $F$ of $N$ must be orientable since $M$ is in $\mathcal{M}$. Let $N_{0}$ denote the complement of tubular neighborhoods of the singular fibers (or one regular fiber if there are no singular ones) of $N$ and let $F_{0}$ denote $F \cap N_{0}$. Then $N_{0}$ is the trivial circle bundle over $F_{0}$ and generators for $\pi_{1} N$ 
are represented by a circle fiber and loops representing generators of $\pi_{1} F_{0}$. Denote by $t \in \pi_{1} N$ the element represented by a regular fiber (a circle fiber of $\left.N_{0}\right)$.

We may assume that $\gamma$ is not represented by a singular fiber because such elements of $\pi_{1}(M)$ have cyclic centralizers.

Consider the case where $\gamma$ is in the cyclic subgroup $\langle t\rangle$ generated by a regular fiber. Since $F$ is orientable, $\langle t\rangle$ is central in $\pi_{1} N$, so in this case $\zeta(\gamma)=\pi_{1} N$. Take $k_{0} \in \mathcal{K}_{\gamma}(M)$ to be contained in a neighborhood of a regular fiber. As in the cyclic centralizer case discussed above, the trace $K_{t}^{0}$ of a longitudinal self-isotopy of $k_{0}$ around the fiber direction of $N_{0}$ has a latitude representing $t$. Any generator $\phi$ of $\pi_{1} N$ that is carried by $F_{0}$ can be represented by a latitude of the trace $K_{\phi}^{0}$ of a self-isotopy of $k_{0}$ that pushes the neighborhood of the fiber containing $k_{0}$ around $N_{0}$ over any loop in $F_{0}$ representing $\phi$.

Now consider the case where $\gamma$ is not carried by any fiber. Then $\zeta(\gamma)$ is isomorphic to $\mathbb{Z} \oplus \mathbb{Z}$ unless $F$ is a closed torus so that $N=T^{3}$ (possibly punctured); this is because $M$ contains no non-vertical tori (and by our assumption that $\pi_{1} M$ is not cyclic). The theorem holds for $M=T^{3}$, since any knot in $T^{3}$ can be self-isotoped around the circle factors which generate $\pi_{1}\left(T^{3}\right) \cong \mathbb{Z} \oplus \mathbb{Z} \oplus \mathbb{Z}$, so we will assume that $F$ is not a closed torus. One of the factors of $\zeta(\gamma)$ is generated by $t$ and the other is generated by a root $\rho$ of $\gamma$. Take $k_{0} \in \mathcal{K}_{\gamma}(M)$ to be contained in a tubular neighborhood of any knot in $N_{0}$ that represents $\rho$. Then the trace $K_{t}^{0}$ of a self-isotopy of $k_{0}$ around the fiber has a latitude representing $t$ and the trace $K_{\rho}^{0}$ of a longitudinal self-isotopy of $k_{0}$ has a latitude representing $\rho$.

\section{Examples}

\subsection{Knots in $\mathrm{S}^{1} \times \mathrm{S}^{2}$}

Consider $M=S^{1} \times S^{2} \in \mathcal{M}$ with $\pi_{1}(M)$ generated (multiplicatively) by $x=$ $\left[S^{1}\right]$ and $\pi_{2}(M)$ generated by $\sigma=\left[S^{2}\right]$. If $\gamma=x^{n}$, then $\left\langle x^{n}\right\rangle$ is normal in $\pi_{1}(M)$ and $\tilde{\Lambda}_{x^{n}}$ is generated by coset representatives $\left\{x, x^{2}, \ldots, x^{n / 2}\right\}$ if $n$ is even, or $\left\{x, x^{2}, \ldots, x^{(n-1) / 2}\right\}$ if $n$ is odd (recall the relations $1=0$ and $g=g^{-1}$ ). Since all knots in $S^{1} \times S^{2}$ can be isotoped around the $S^{1}$ direction using the product structure, any $k$ in $\mathcal{K}_{x^{n}}\left(S^{1} \times S^{2}\right)$ is spherical. Since $\pi_{1} M$ is abelian, conjugation actions are trivial so $\left(\tilde{\Lambda}_{x^{n}} /\left(\Phi(k), \zeta\left(x^{n}\right)\right)=\tilde{\Lambda}_{x^{n}} / \Phi(k)\right.$. By Proposition 5.4.1, we see that the generators of $\Phi(k)$ are $\left(x^{m} \lambda(\sigma, k), 1\right)=\left(x^{m}\left(1+x+x^{2}+\cdots+\right.\right.$ 


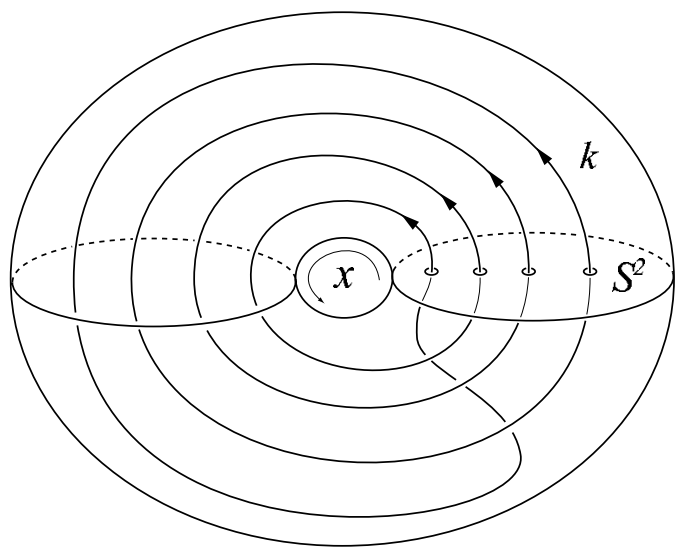

Figure 10

$\left.x^{n-1}\right), 1$ ) as can be computed from Figure 10 (showing a knot $k$ in $\mathcal{K}_{x^{4}}\left(S^{1} \times\right.$ $\left.S^{2}\right)$ ). So in this case the target orbit space $\tilde{\Lambda}_{x^{n}} / \Phi(k)$ is a group, the quotient of the free abelian group $\mathbb{Z}\left[x, x^{2}, \ldots, x^{n / 2}\right]$ or $\mathbb{Z}\left[x, x^{2}, \ldots, x^{(n-1) / 2}\right]$ by a single relation $x^{m}\left(1+x+x^{2}+\cdots+x^{n-1}\right)=x+x^{2}+\cdots+x^{n-1}=0$. (This computation can be used to affirm the conjecture at the end of [19].) Note that if $n$ is odd, then this relation introduces a 2-torsion element:

$$
x+x^{2}+\cdots+x^{n-1}=2\left(x+x^{2} \cdots+x^{(n-1) / 2}\right)=0
$$

and if $n$ is even, the relation eliminates the middle degree generator:

$$
x^{n / 2}=-2\left(x+x^{2}+\cdots+x^{(n / 2)-1}\right) .
$$

For instance, if $n=1$ or $n=2$, then the target space is trivial (and any two knots in $\mathcal{K}_{x}\left(S^{1} \times S^{2}\right)$ or in $\mathcal{K}_{x^{2}}\left(S^{1} \times S^{2}\right)$ are $W$-equivalent).

If $n=3$, then the target space is $\mathbb{Z}_{2}$ since $2 x=0$.

For $n=4$, the relation $x^{2}=-2 x$ reduces the target space to $\mathbb{Z}$ (generated by the coefficient of $x$ ). For example, the knot $j$ pictured in Figure 11 is gotten from the knot $k$ of Figure 10 by three crossing changes (labelled $p_{1}, p_{2}$ and $p_{3}$ ) of the same sign which describe the trace $H$ of a homotopy from $k$ to $j$ with three singularities. Computing the corresponding group elements yields $\mu_{k}(j)=\mu(H)=x+x+x^{2}$ which vanishes in the target space. We conclude that $k$ and $j$ are $W$-equivalent. A singular concordance admitting Whitney disks can be constructed by tubing an $S^{2}$ into $H$ and performing a cusp homotopy. 


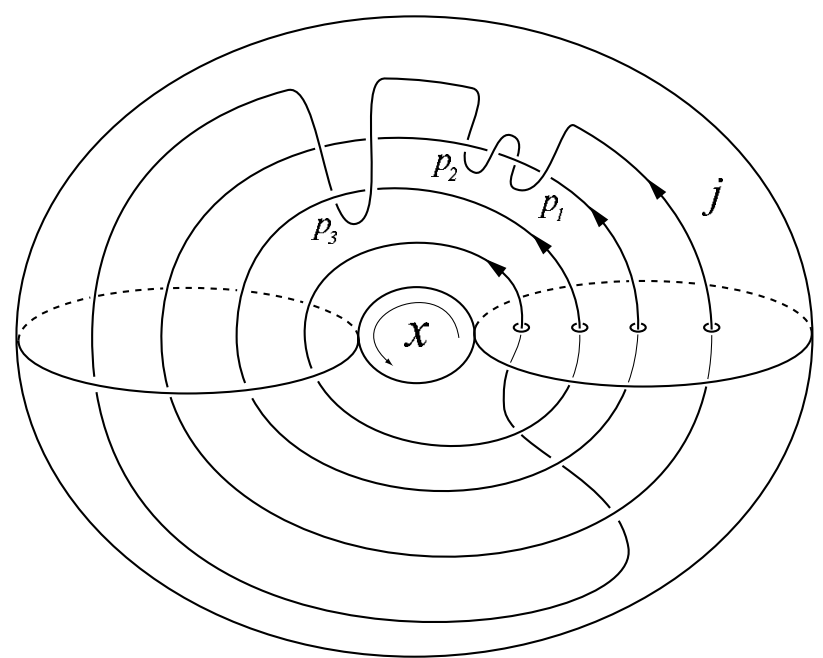

Figure 11

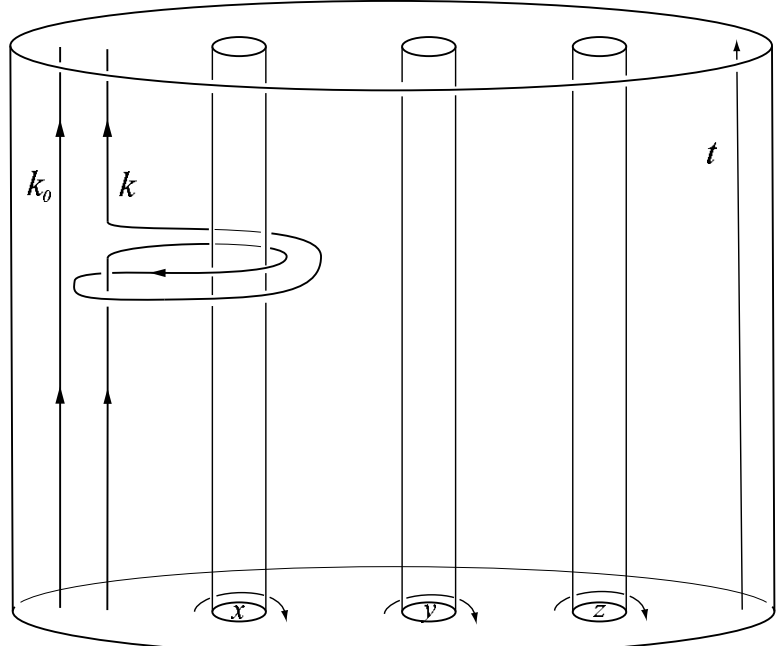

Figure 12: A spherical knot $k_{0}$ and a non-spherical knot $k$

\subsection{Non-spherical example}

Illustrated in Figure 12 are two knots $k_{0}$ and $k$ in $\mathcal{K}_{t}(M)$ where $M \in \mathcal{M}$ is the product $F \times S^{1}$ of a thrice punctured disk $F$ with the circle and $t \in \pi_{1}(M)=$ $\langle x, y, z\rangle \times\langle t\rangle$ is represented by a circle fiber. As described in the proof of Theorem $4, k_{0}$ can be self-isotoped around each generator of $\zeta(t)=\pi_{1} M$ so $k_{0}$ 


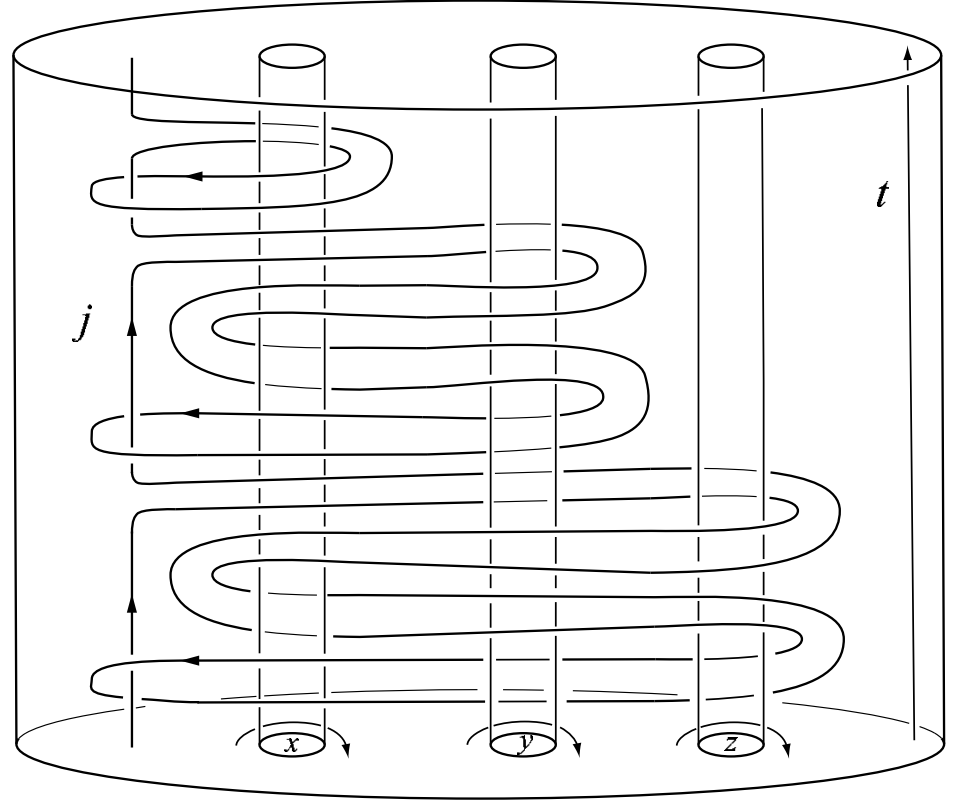

Figure 13

is spherical. Since $M$ is irreducible, the action of $\Phi\left(k_{0}\right)$ reduces to conjugation (by Proposition 5.4.1) and the target space for relative self-linking numbers with respect to $k_{0}$ is the orbit space $\widetilde{\Lambda}_{t} /\left(\pi_{1} M\right)$ of $\widetilde{\Lambda}_{t}$ under conjugation by $\zeta(t)=\pi_{1} M$. Computing with the trace $H_{0}$ of the obvious homotopy (through one clasp singularity) from $k_{0}$ to $k$ yields $\mu_{k_{0}}(k)=x \neq 0 \in \widetilde{\Lambda}_{t} /\left(\pi_{1} M\right)$ (for some fixed orientation convention). If $K_{0}$ is the trace of any self-isotopy of $k_{0}$ with a latitude determining $\alpha \neq 1$, then $K_{\alpha}:=-H_{0}+K_{0}+H_{0}$ is the trace of a self-homotopy of $k$ having a latitude that determines $\alpha$ such that

$$
\mu\left(K_{\alpha}\right)=-x+\alpha x \alpha^{-1} \neq 0 \in \widetilde{\Lambda}_{t} .
$$

(Here we are using the same whisker for $k_{0}$ and $k$ as well as assuming that latitudes of $H_{0}$ are trivial.) We conclude that $k$ is not spherical (by Lemma 5.3.1 and the irreducibility of $M$ ).

Consider now the knot $j \in \mathcal{K}_{t}(M)$ pictured in Figure 13. Using the trace $J_{0}$ of the obvious homotopy (through 3 clasp singularities) from $k_{0}$ to $j$, one computes $\mu_{k_{0}}(j)=x+y x y^{-1}-y z x(y z)^{-1} \in \widetilde{\Lambda}_{t} /\left(\pi_{1} M\right)$. By Theorem $3, j$ is not $W$-equivalent to $k$ since $\mu_{k_{0}}(j) \neq \mu_{k_{0}}(k) \in \widetilde{\Lambda}_{t} /\left(\pi_{1} M\right)$, that is, since $\mu\left(J_{0}\right)=x+y x y^{-1}-y z x(y z)^{-1} \in \widetilde{\Lambda}_{t}$ is not conjugate to $\mu\left(H_{0}\right)=x \in \widetilde{\Lambda}_{t}$.

Computing with the trace $H$ of the obvious homotopy (through 2 clasp singu- 
larities) from $k$ to $j$ yields

$$
\begin{aligned}
\mu_{k}(j)=\mu(H) & =-y x y^{-1}+(y z) x(y z)^{-1} \\
& =y\left(-x+z x z^{-1}\right) y^{-1} \\
& =y\left(\left(\mu\left(K_{z}\right), z\right) \cdot 0\right) y^{-1} \\
& =0 \in \widetilde{\Lambda}_{t} /\left(\pi_{1} M\right) .
\end{aligned}
$$

where $K_{z}$ is the trace of a self-homotopy of $k$ as in $\left({ }^{* *}\right)$ above. Thus, $\mu_{k}(j)$ vanishes even though $k$ and $j$ are not $W$-equivalent, illustrating the necessity of spherical knots in the hypotheses of Theorem 3.

\subsection{A family of 3-component links}

Here we consider knots in the complement $M$ of a 2 -component unlink $k_{1} \cup k_{2}$ in $S^{3}$, that is, $M=S^{1} \times D^{2} \sharp S^{1} \times D^{2} \in \mathcal{M}$ with $\pi_{1} M=\langle x, y\rangle$ free on two generators represented by meridians to $k_{1} \cup k_{2}$. Any splitting 2-sphere for the unlink represents a generator $\sigma$ of $\pi_{2} M$ as a module over $\langle x, y\rangle$.

If $\gamma=\prod_{i=1}^{r} x^{m_{i}} y^{n_{i}} \in \pi_{1} M$, then, for any $k \in \mathcal{K}_{\gamma}(M), \widetilde{\lambda}(\sigma, k)$ is equal to

$$
\begin{gathered}
1-\left(x^{m_{1}}\right)^{-1}+\left(x^{m_{1}} y^{n_{1}}\right)^{-1}-\left(x^{m_{1}} y^{n_{1}} x^{m_{2}}\right)^{-1}+\ldots \\
\ldots+\left(x^{m_{1}} y^{n_{1}} \cdots x^{m_{r-1}} y^{n_{r-1}}\right)^{-1}-\left(x^{m_{1}} y^{n_{1}} \cdots x^{m_{r}}\right)^{-1} \in \widetilde{\Lambda}_{\gamma} .
\end{gathered}
$$

Since $\pi_{1} M$ is free, there exist spherical knots in every homotopy class since $\zeta(\gamma)$ is cyclic for all $\gamma \in \pi_{1} M$ (Remark 7.2.1). In the case that $\gamma$ is primitive, then every knot $k$ in $\mathcal{K}_{\gamma}(M)$ is spherical and the conjugation action of $\zeta(\gamma)=\langle\gamma\rangle$ on $\widetilde{\Lambda}_{\gamma}$ is trivial so relative self-linking with respect to $k$ maps $\mathcal{C}_{\gamma}(M)$ (and $W$ equivalence classes) onto the group generated by $\widetilde{\Lambda}_{\gamma}$ modulo the infinite family of relations $\tilde{\lambda}(g \cdot \sigma, k)=g \widetilde{\lambda}(\sigma, k)=0$, where $g$ ranges over $\pi_{1} M$.

For example, if $\gamma=x y$, then we have the relations $g \widetilde{\lambda}(\sigma, k)=g-g x^{-1}=$ $g-g y=0 \in \widetilde{\Lambda}_{x y}$, and one can check (for instance, by induction on the length of reduced double coset representatives) that in this case $\widetilde{\Lambda}_{x y} / \Phi(k)=0$ so that any two knots in $\mathcal{K}_{x y}(M)$ are $W$-equivalent.

The case $\gamma=x^{2} y$ shows that relative self-linking numbers are not in general determined by projecting to the irreducible factors of $M$ : Here the relations are $g-g x^{-2}=g-g y=0 \in \widetilde{\Lambda}_{x^{2} y} / \Phi(k)$. One can check that $x y x^{-1}$ is non-zero in $\widetilde{\Lambda}_{x^{2} y} / \Phi(k)$ (which is infinitely generated) and pictured in Figure 14 are knots $k$ and $j$ in $\mathcal{K}_{x^{2} y}(M)$ with $\mu_{k}(j)=x y x^{-1} \neq 0$. We conclude that $k$ and $j$ are not $W$-equivalent, a fact that is not detected by the quotient relative self-linking numbers that ignore either one of the components of $k_{1} \cup k_{2}$ and which take 


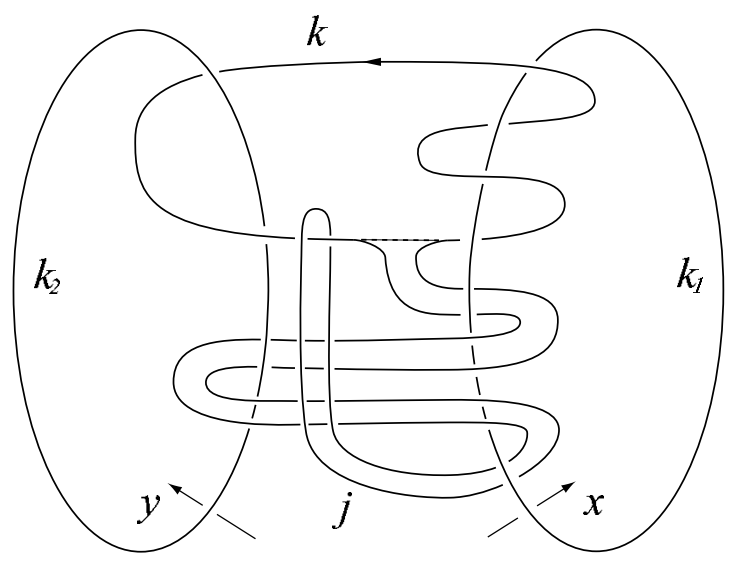

Figure 14: The dotted arc corresponds to the knot $k$ and the "finger" corresponds to the knot $j$.

values in the trivial group and $\mathbb{Z}_{2}$ respectively. (In fact, the links $k \cup k_{i}$ and $j \cup k_{i}$ are isotopic in $S^{3}$ for $i=1,2$.) This illustrates the "non-abelian" nature of relative self-linking numbers.

A more complex example is given by $\gamma=[x, y]:=x y x^{-1} y^{-1}$ (which includes the case where $k$ is one component of the Borromean rings). Here the target group for relative self-linking numbers with respect to any $k \in \mathcal{K}_{[x, y]}(M)$ is described by the relations

$$
g-g x^{-1}+g x^{-1} y^{-1}-g y^{-1}=0 \in \widetilde{\Lambda}_{[x, y]} / \Phi(k)
$$

where $g$ ranges over $\langle x, y\rangle$.

One can check that

$$
x y=y^{-1} x^{-1}=y x=x^{-1} y^{-1}=x y^{-1}=y x^{-1}=x+y
$$

in $\widetilde{\Lambda}_{[x, y]} / \Phi(k)$ which appears to "almost" split apart all cross terms; however, I conjecture that

$$
x^{-1} y=y^{-1} x \neq x \pm y
$$

in $\widetilde{\Lambda}_{[x, y]} / \Phi(k)$ and that some cross term generators cannot be eliminated. (A "reason" for this might have to do with the fact that $x^{-1} y$ and $y^{-1} x$ do not occur as sub-words of $\gamma$ or $\gamma^{-1}$.) It would be nice to have algebraic techniques to handle what appears to be a subtly non-abelian case.

It is worth noting that projecting to the free Milnor group (which adds the relations $\left[x, x^{g}\right]=1=\left[y, y^{g}\right], g \in\langle x, y\rangle$ ) centralizes $[x, y]$, and the resulting quotient of relative self-linking numbers is indeed determined by its (abelian) 
projections to the irreducible sub-manifolds and its vanishing implies that the 3-component links $k \cup k_{1} \cup k_{2}$ and $j \cup k_{1} \cup k_{2}$ are in fact disjointly $W$-equivalent, meaning that their components co-bound in $S^{3} \times I$ disjoint properly immersed annuli admitting Whitney disks. It is clear that relative self-linking numbers give isotopy invariants of classical links; a study of the more subtle question of connections with classical link concordance invariants would be interesting.

Remark 8.3.1 The examples of 8.3 illustrate that computing in the infinitely presented groups $\widetilde{\Lambda}_{\gamma} / \Phi(k)$ is an interesting algebraic problem from a topological point of view: On the one hand, the data is given purely in algebraic terms and on the other hand, concordance classes of knots map onto the groups which are generated by double coset spaces which themselves correspond to intersection invariants of non-simply-connected manifolds.

\section{$9 \quad 2-$-component links}

This section describes absolute and relative linking numbers for 2-component links which are straightforward analogues of the self-linking and relative selflinking numbers previously defined for knots. A generalized intersection invariant $\lambda$ for immersed annuli plays the role of the generalized Wall self-intersection invariant $\mu$ and indeterminacies are factored out in exactly the same way as for knots with analogous results in many cases. Since the techniques and arguments involved are so similar, most proofs and details will be omitted.

For links of null-homotopic knots, the (absolute) algebraic linking number $\lambda$ will play a role in defining a connected sum operation for null-homotopic knots that yields a group structure on certain equivalence classes of null-homotopic knots (9.1.3).

Two other points worth noting are the geometric characterization of relative linking with respect to a spherical link in terms of disjointness (9.7.2) and the modifications of Theorem 4 needed to formulate an analogous theorem for links (Theorem 7) as illustrated in Example 9.7.3.

Definition 9.0.2 Let $\mathcal{L}_{\gamma, \delta}(M)$ denote the set of two component links $k_{1} \cup k_{2}$ with $k_{1} \in \mathcal{K}_{\gamma}(M)$ and $k_{2} \in \mathcal{K}_{\delta}(M)$. Let $\mathcal{C}_{\gamma, \delta}(M)$ denote $\mathcal{L}_{\gamma, \delta}(M)$ modulo link concordance. 


\subsection{Links of null-homotopic knots}

We first consider 2-component links of null-homotopic knots.

Definition 9.1.1 For any $M$ and any link $k_{1} \cup k_{2} \in \mathcal{L}_{1,1}(M)$, define the (algebraic) linking number $\lambda\left(k_{1} \cup k_{2}\right)$ of $k_{1} \cup k_{2}$ by

$$
\lambda\left(k_{1} \cup k_{2}\right):=\lambda\left(D_{1}, D_{2}\right) \in \Lambda=\mathbb{Z}\left[\pi_{1} M\right]
$$

where $\lambda\left(D_{1}, D_{2}\right)$ is the intersection number (2.1) of any properly immersed 2-disks $D_{1}$ and $D_{2}$ in $M \times I$ bounded by $k_{1} \cup k_{2} \subset M \times\{0\}$.

The proof of the following theorem follows directly from the arguments in Section 3 .

Theorem 5 For any oriented 3-manifold $M$, algebraic linking numbers induce a well-defined map from $\mathcal{C}_{1,1}(M)$ onto $\Lambda$.

Since absolute linking invariants contain no new indeterminacies, their geometric properties are inherited directly from Wall's intersection invariant (2.1); as a corollary we get that absolute linking and self-linking numbers characterize disjoint $W$-equivalence to the unlink:

Corollary 9.1.2 For any $M$ and any link $k_{1} \cup k_{2} \in \mathcal{L}_{1,1}(M)$, the following are equivalent:

(i) The self-linking numbers $\mu\left(k_{1}\right), \mu\left(k_{2}\right)$ and the linking number $\lambda\left(k_{1} \cup k_{2}\right)$ all vanish.

(ii) There exist disjoint properly immersed disks $D_{1}$ and $D_{2}$ in $M \times I$ bounded by $k_{1} \cup k_{2}$ in $M \times\{0\}$ with Whitney disks pairing all self-intersections of $D_{1}$ and $D_{2}$.

Proof Corollary 9.1.2 follows directly from Proposition 2.1.3. The comment before the statement of Corollary 9.1.2 regarding disjoint $W$-equivalence to the unlink is confirmed by observing that given properly immersed disks in $M \times I$ bounded by a link in $M \times\{0\}$, one can construct a singular concordance to the unlink by removing a small open disk from each immersed disk followed by an isotopy $($ rel $\partial)$. 


\subsubsection{An equivalence relation on $\mathcal{K}_{1}(\mathrm{M})$ admitting a group structure}

The notion of $W$-equivalence corresponds to the singularities of a singular concordance coming in cancelling pairs with null-homotopic Whitney circles ([7]). If we weaken this notion by only requiring that the Whitney circles bound tori (rather than disks) then the resulting equivalence relation (for knots) is characterized by a slightly weaker absolute self-linking number $\mu^{\pi}(k)$ which takes values in the free abelian group $\widetilde{\Lambda}^{\pi}$ generated by the non-trivial conjugacy classes of $\pi_{1} M$ modulo inversion (a quotient of $\widetilde{\Lambda}$ ). The resulting equivalence classes $\mathcal{K}_{1}^{\pi}(M)$ in $\mathcal{K}_{1}(M)$ can be made into a group:

Corollary 9.1.3 There exists a connected sum operation $\sharp$ that makes $\mathcal{K}_{1}^{\pi}(M)$ into a group isomorphic to $\widetilde{\Lambda}^{\pi}$, the isomorphism being given by $\mu^{\pi}$.

Proof The connected sum operation $\sharp$ of Corollary 9.1 .3 will be defined after giving the precise definition of $\mu^{\pi}(k)$. The usual definition of connected sum for knots in $S^{3}$ (which makes $\mathcal{C}\left(S^{3}\right)$ into an abelian group) involves two steps; first the knots are separated by any embedded 2 -sphere, then they are joined by the (orientation-preserving) band sum (0-surgery) along any choice of band that meets the 2-sphere in a standard way. Here we first achieve an "algebraic separation" by requiring the vanishing of the absolute algebraic linking number, then join the knots along any generic band.

Define $\widetilde{\Lambda}^{\pi}$ by

$$
\widetilde{\Lambda}^{\pi}:=\frac{\mathbb{Z}\left[\pi_{1} M\right]}{\mathbb{Z}[1] \oplus\left\langle g-x g^{ \pm 1} x^{-1}\right\rangle} ; \quad x, g \in \pi_{1} M
$$

that is, $\widetilde{\Lambda}^{\pi}$ is the free abelian group generated by the non-trivial conjugacy classes of $\pi_{1} M$ modulo inversion. ( $\widetilde{\Lambda}^{\pi}$ is the quotient of $\widetilde{\Lambda}$ by the relations $g-x g x^{-1}=0$ for $x, g \in \pi_{1} M$.)

Definition 9.1.4 For $k \in \mathcal{K}_{1}(M)$, define

$$
\mu^{\pi}(k):=\mu(D) \in \widetilde{\Lambda}^{\pi}
$$

where $D$ is any singular null-concordance for $k$.

If $\mu^{\pi}(k)=\mu^{\pi}(j)$ we say that $k$ and $j$ are $\mu^{\pi}$-equivalent. Denote by $\mathcal{K}_{1}^{\pi}(M)$ the set $\mathcal{K}_{1}(M)$ modulo $\mu^{\pi}$-equivalence.

It follows from Theorem 3 (and the fact that the unknot is spherical) that two knots are $\mu^{\pi}$-equivalent if and only if they co-bound a singular concordance 
with all singularities paired by "Whitney tori" (rather than disks) since the singularities will occur in cancelling pairs with null-homologous Whitney-circles (rather than null-homotopic) due to the relations $g-x g x^{-1}=0$ added in the definition of $\mu^{\pi}(k)$.

For knots $k_{1}$ and $k_{2}$ in $\mathcal{K}_{1}^{\pi}(M)$ define $k_{1} \sharp k_{2}$ as follows:

First perturb $k_{1}$ to be disjoint from $k_{2}$ in $M$, then isotope $k_{1}$ so that $\lambda\left(k_{1}, k_{2}\right)=$ $0 \in \Lambda$. This isotopy can be assumed to be supported in the neighborhood of a finite collection of arcs which guide crossing changes between $k_{1}$ and $k_{2}$. Of course this isotopy does not change the class of $k_{1}$ or $k_{2}$ in $\mathcal{K}_{1}^{\pi}(M)$ (or in $\left.\mathcal{K}_{1}(M)\right)$.

Now define $k_{1} \sharp k_{2}$ to be the usual orientation preserving band sum (0-surgery) of $k_{1}$ with $k_{2}$ along any band $b$ (whose interior is disjoint from $k_{1} \cup k_{2}$ ).

If $D_{1}$ and $D_{2}$ are any singular null-concordances of $k_{1}$ and $k_{2}$, then the band sum $D_{1} \sharp_{b} D_{2}$ is a singular null-concordance for $k_{1} \sharp k_{2}$. Taking the whisker on $D_{1}$ as a whisker for $D_{1} \sharp_{b} D_{2}$ we have

$$
\mu\left(D_{1} \sharp_{b} D_{2}\right)=\mu\left(D_{1}\right)+\beta \mu\left(D_{2}\right) \beta^{-1}+\lambda\left(k_{1}, k_{2}\right) \beta^{-1} \in \widetilde{\Lambda}
$$

where $\beta \in \pi_{1} M$ is determined by the band $b$ and the whiskers on $k_{1}$ and $k_{2}$. Since $\lambda\left(k_{1}, k_{2}\right)$ vanishes, taking this equation in $\widetilde{\Lambda}^{\pi}$ yields

$$
\mu^{\pi}\left(k_{1} \sharp k_{2}\right)=\mu^{\pi}\left(k_{1}\right)+\mu^{\pi}\left(k_{2}\right)
$$

which shows that $\sharp$ is well-defined on $\mathcal{K}_{1}^{\pi}(M)$. Inverses are gotten by reversing knot orientations; $\mu^{\pi}$ is injective by definition and surjective by Theorem 5 .

\subsection{Links of essential knots}

The definition of relative linking numbers for links of essential knots follows directly along the lines of the case of relative self-intersection numbers for knots. The rest of this section contains statements of the analogous theorems without proofs.

\subsection{Intersection numbers for annuli}

Let $A$ and $B$ be a pair of properly immersed annuli in a 4 -manifold $X$. Chose whiskers for each of $A$ and $B$ and let $\langle\gamma\rangle$ and $\langle\delta\rangle$ be the respective cyclic images in $\pi_{1} X$ of the induced maps on fundamental groups. The group element associated to an intersection point from a loop through $A$ and back through $B$ 
in the usual way is only well-defined up to left multiplication by powers of $\gamma$ and right multiplication by powers of $\delta$. Thus, we work with the double cosets of $\pi_{1} X$ by $\langle\gamma\rangle$ on the left and $\langle\delta\rangle$ on the right. Adjusting the notation of 4.1, we define

$$
\Lambda_{\gamma, \delta}:=\mathbb{Z}\left[\langle\gamma\rangle \backslash \pi_{1} X /\langle\delta\rangle\right]=\frac{\mathbb{Z}\left[\pi_{1} X\right]}{\left\{g-\gamma^{n} g \delta^{m}\right\}}
$$

where $n$ and $m$ range over the integers.

Definition 9.3.1 Let $A$ and $B$ be properly immersed annuli (equipped with whiskers) in a 4 -manifold $X$ whose fundamental groups map to $\langle\gamma\rangle$ and $\langle\delta\rangle$ respectively. The intersection number $\lambda(A, B)$ of $A$ and $B$ is defined by

$$
\lambda(A, B):=\sum(\operatorname{sign} p) \cdot g_{p} \in \Lambda_{\gamma, \delta}
$$

where the sum is over all intersection points $p \in A \cap B$ and $\operatorname{sign} p$ comes from the orientations of $X$ and the sheets of $A$ and $B$ at $p$ as usual.

Note that $\mu(A, B)$ is well-defined up to left (resp. right) multiplication by elements of $\zeta(\gamma)$ (resp. $\zeta(\delta)$ ) corresponding to the effect of changing the whisker of $A$ (resp. $B$ ) while preserving the image of the induced map on fundamental groups.

A whisker change that does not preserve the cyclic images also changes the target space by an isomorphism.

Proposition 9.3.2 The intersection number $\lambda(A, B) \in \Lambda_{\gamma, \delta}$ is invariant under homotopy (rel $\partial$ ) and vanishes if and only if there exists Whitney disks pairing all intersections between $A$ and $B$ if and only if $A$ and $B$ can be homotoped (rel $\partial$ ) to be disjoint.

\subsection{The indeterminacy subgroup $\Phi\left(k_{1} \cup k_{2}\right)$}

Definition 9.4.1 For any link $k_{1} \cup k_{2} \in \mathcal{L}_{\gamma, \delta}(M)$, define

$$
\Phi\left(k_{1} \cup k_{2}\right) \leq \Lambda_{\gamma, \delta} \rtimes(\zeta(\gamma) \times \zeta(\delta))
$$

to be the subgroup of the semi-direct product of $\Lambda_{\gamma, \delta}$ and $\zeta(\gamma) \times \zeta(\delta)$ with respect to the action

$$
(\phi, \psi): y \mapsto \phi y \psi^{-1}
$$


of $\zeta(\gamma) \times \zeta(\delta)$ on $\Lambda_{\gamma, \delta}$ generated by the elements

$$
\left(\lambda\left(K_{1}, K_{2}\right)\left(w_{1}, w_{2}\right),\left(\operatorname{lat}\left[K_{1}\right]\left(w_{1}\right), \operatorname{lat}\left[K_{2}\right]\left(w_{2}\right)\right)\right.
$$

as $K_{1} \cup K_{2}$ ranges over all singular self-concordances of $k_{1} \cup k_{2}$ and $w_{1}$ (resp. $\left.w_{2}\right)$ ranges over all whiskers identifying $\left[k_{1}\right]=\gamma \in \pi_{1} M\left(\operatorname{resp} .\left[k_{2}\right]=\delta \in \pi_{1} M\right)$.

\subsubsection{The action of $\Phi\left(k_{1} \cup k_{2}\right)$ on $\Lambda_{\gamma, \delta}$}

The group $\Lambda_{\gamma, \delta} \rtimes(\zeta(\gamma) \times \zeta(\delta))$ acts on $\Lambda_{\gamma, \delta}$ by

$$
(z,(\phi, \psi)): y \mapsto z+\phi y \psi^{-1}
$$

for $(z,(\phi, \psi)) \in \Lambda_{\gamma, \delta} \rtimes(\zeta(\gamma) \times \zeta(\delta))$ and $y \in \Lambda_{\gamma, \delta}$. We denote the equivalence classes under the restriction of this action to the subgroup $\Phi\left(k_{1} \cup k_{2}\right)$ by

$$
\Lambda_{\gamma, \delta} / \Phi\left(k_{1} \cup k_{2}\right) .
$$

\subsubsection{The action of $\zeta(\gamma) \times \zeta(\delta)$ on $\Lambda_{\gamma, \delta} / \Phi\left(k_{1} \cup k_{2}\right)$}

The effect that changing the whiskers for $k_{1} \cup k_{2} \in \mathcal{L}_{\gamma, \delta}(M)$ has on a generator of $\Phi\left(k_{1} \cup k_{2}\right)$ is described by an action of $\zeta(\gamma) \times \zeta(\delta)$ on $\Lambda_{\gamma, \delta} / \Phi\left(k_{1} \cup k_{2}\right)$ defined by:

$$
(\alpha, \beta):(z,(\phi, \psi)) \mapsto\left(\alpha z \beta^{-1},\left(\alpha \phi \alpha^{-1}, \beta \psi \beta^{-1}\right)\right)
$$

for all $(\alpha, \beta) \in \zeta(\gamma) \times \zeta(\delta)$.

We have a well-defined conjugation action of $\zeta(\gamma) \times \zeta(\delta)$ on $\Lambda_{\gamma, \delta} / \Phi\left(k_{1} \cup k_{2}\right)$ since if

$$
x=z+\phi y \psi^{-1} \in \Lambda_{\gamma, \delta}
$$

we have

$$
\alpha x \beta^{-1}=\alpha z \beta^{-1}+\left(\alpha \phi \alpha^{-1}\right)\left(\alpha y \beta^{-1}\right)\left(\beta \psi^{-1} \beta^{-1}\right) \in \Lambda_{\gamma, \delta} .
$$

Denote by

$$
\Lambda_{\gamma, \delta} /\left(\Phi\left(k_{1} \cup k_{2}\right), \zeta(\gamma) \times \zeta(\delta)\right)
$$

the orbit space of $\Lambda_{\gamma, \delta} / \Phi\left(k_{1} \cup k_{2}\right)$ under the action of $\zeta(\gamma) \times \zeta(\delta)$. 


\subsection{Definition of relative linking numbers $\lambda_{k_{1} \cup k_{2}}\left(j_{1} \cup j_{2}\right)$}

Definition 9.5.1 Fix a link $k_{1} \cup k_{2}$ in $\mathcal{L}_{\gamma, \delta}(M)$. For all $j_{1} \cup j_{2} \in \mathcal{L}_{\gamma, \delta}(M)$, define $\lambda_{k_{1} \cup k_{2}}\left(j_{1} \cup j_{2}\right)$, the relative linking number of $j_{1} \cup j_{2}$ with respect to $k_{1} \cup k_{2}$, by

$$
\lambda_{k_{1} \cup k_{2}}\left(j_{1} \cup j_{2}\right):=\lambda\left(H_{1}, H_{2}\right) \in \Lambda_{\gamma, \delta} /\left(\Phi\left(k_{1} \cup k_{2}\right), \zeta(\gamma) \times \zeta(\delta)\right)
$$

where $H_{1} \cup H_{2}$ is any singular concordance from $k_{1} \cup k_{2}$ to $j_{1} \cup j_{2}$.

Theorem 6 For each $k_{1} \cup k_{2} \in \mathcal{L}_{\gamma, \delta}(M)$, the map $j_{1} \cup j_{2} \mapsto \lambda_{k_{1} \cup k_{2}}\left(j_{1} \cup j_{2}\right)$ induces a well-defined map

$$
\mathcal{C}_{\gamma, \delta}(M) \rightarrow \Lambda_{\gamma, \delta} /\left(\Phi\left(k_{1} \cup k_{2}\right), \zeta(\gamma) \times \zeta(\delta)\right)
$$

onto the target space.

\subsection{A generating set for $\Phi\left(k_{1} \cup k_{2}\right)$}

Proposition 9.6.1 Given $k_{1} \cup k_{2} \in \mathcal{L}_{\gamma, \delta}(M)$, let $\left\{\phi_{q}\right\}$ (resp. $\left\{\psi_{r}\right\}$ ) be any generating set for the centralizer $\zeta(\gamma)$ (resp. $\zeta(\delta))$ and let $\left\{K_{q}^{1}\right\}$ (resp. $\left\{K_{r}^{2}\right\}$ ) be a corresponding set of traces of self-homotopies of $k_{1}$ (resp. $k_{2}$ ) such that, for a fixed whisker $w_{1}$ on $k_{1}$ (resp. $w_{2}$ on $k_{2}$ ), lat $\left[K_{q}^{1}\right]\left(w_{1}\right)=\phi_{q}$ for all $q$ (resp. lat $\left[K_{r}^{2}\right]\left(w_{2}\right)=\psi_{r}$ for all $\left.r\right)$. Denote the traces $k_{1} \times I$ and $k_{2} \times I$ of constant self-homotopies of $k_{1}$ and $k_{2}$ by $K^{1}$ and $K^{2}$. Let $\left\{\sigma_{s}\right\}$ be any generating set for $\pi_{2} M$ as a module over $\pi_{1} M$.

Let $\mathcal{S}_{0} \subset \Lambda_{\gamma, \delta} \rtimes(\zeta(\gamma) \times \zeta(\delta))$ denote the union of the sets

$$
\left\{\left(\lambda\left(K_{q}^{1}, K^{2}\right)\left(w_{1}, w_{2}\right),\left(\phi_{q}, 1\right)\right),\left(\lambda\left(K^{1}, K_{r}^{2}\right)\left(w_{1}, w_{2}\right),\left(1, \psi_{r}\right)\right),\right\}
$$

and

$$
\left\{\left(g \lambda\left(\sigma_{s}, k_{2}\right)\left(w_{1}, w_{2}\right),(1,1)\right),\left(\lambda\left(k_{1}, \sigma_{s}\right)\left(w_{1}, w_{2}\right) g,(1,1)\right)\right\}
$$

where $g$ ranges over $\pi_{1} M$.

Then $\Phi\left(k_{1} \cup k_{2}\right)$ is generated by

$$
\bigcup_{(\alpha, \beta) \in \zeta(\gamma) \times \zeta(\delta)} \alpha \mathcal{S}_{0} \beta^{-1}
$$

that is, by the union of all the sets in the orbit of $\mathcal{S}_{0}$ under the action of $\zeta(\gamma) \times \zeta(\delta)$. 


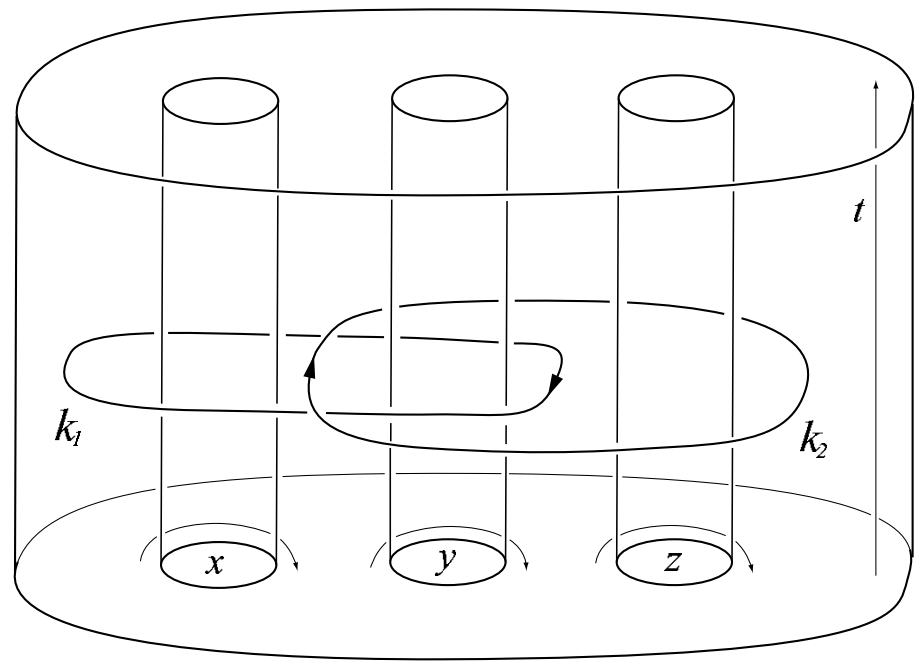

Figure 15: A non-spherical link in the product of a thrice punctured 2-disk with the circle

\subsection{Spherical links}

Definition 9.7.1 A link $k_{1} \cup k_{2} \in \mathcal{L}_{\gamma, \delta}(M)$ is spherical if there exist whiskers $w_{1}$ and $w_{2}$ on $k_{1}$ and $k_{2}$ such that for any $(\phi, \psi) \in \zeta(\gamma) \times \zeta(\delta)$ there exist singular self-concordances $K_{\phi}^{1}$ of $k_{1}$ and $K_{\psi}^{2}$ of $k_{2}$ with $\lambda\left(K_{\phi}^{1}, K_{\psi}^{2}\right)=0 \in \Lambda_{\gamma, \delta}$ and lat $\left[K_{\phi}^{1}\right]\left(w_{1}\right)=\phi$ and lat $\left[K_{\psi}^{2}\right]\left(w_{2}\right)=\psi$.

Proposition 9.7.2 If $k_{1} \cup k_{2} \in \mathcal{L}_{\gamma, \delta}(M)$ is spherical, then for any links $j_{1} \cup j_{2}$ and $j_{1}^{\prime} \cup j_{2}^{\prime}$ in $\mathcal{L}_{\gamma, \delta}(M)$ the following are equivalent:

(i) $\lambda_{k_{1} \cup k_{2}}\left(j_{1} \cup j_{2}\right)=\lambda_{k_{1} \cup k_{2}}\left(j_{1}^{\prime} \cup j_{2}^{\prime}\right)$.

(ii) There exists a singular concordance $H^{1} \cup H^{2}$ between $j_{1} \cup j_{2}$ and $j_{1}^{\prime} \cup j_{2}^{\prime}$ such that $H^{1} \cap H^{2}=\emptyset$.

Spherical links are "less plentiful" than spherical knots: Even when restricting to manifolds in $\mathcal{M}$ (Section 7), there may exist pairs $\gamma, \delta$ of elements in $\pi_{1} M$ with $\mathcal{L}_{\gamma, \delta}(M)$ containing no spherical links as described in Example 9.7.3 below.

We do, however, have the following result which says roughly that if the centralizers of $\gamma$ and $\delta$ are either "small enough" or "large enough" then $\mathcal{L}_{\gamma, \delta}(M)$ does contain spherical links. 
Theorem 7 For any $M$, there exists a spherical link in $\mathcal{L}_{\gamma, \delta}(M)$ if any one of the following is satisfied:

(i) Both of the centralizers $\zeta(\gamma)$ and $\zeta(\delta)$ are cyclic.

(ii) Both of $\gamma$ and $\delta$ are carried by regular fibers of the characteristic Seifert fibered sub-manifolds which carry $\zeta(\gamma)$ and $\zeta(\delta)$.

(iii) At least one of $\gamma$ and $\delta$ are equal to $1 \in \pi_{1} M$.

Theorem 7 can be proved along the lines of the proof of Theorem 4 by finding disjoint self-isotopies of the spherical knots described there whose latitudes represent generators of $\zeta(\gamma) \times \zeta(\delta)$.

Example 9.7.3 For $M=F \times S^{1}$ as in Example 8.2, if $\gamma=x y$ and $\delta=y z$, then $\mathcal{L}_{\gamma, \delta}(M)$ contains no spherical links. As illustrated in Figure 15, one can see an obstruction measured by an intersection number between curves in $F$ representing $\gamma$ and $\delta$ which can be used to compute the indeterminacy subgroup $([24])$.

\section{References}

[1] A. Casson and D. Jungreis. Convergence groups and Seifert fibered 3-manifolds. Inventiones Math. Vol. 118, (1994) 441-456.

[2] T. Cochran and P. Melvin. Finite type invariants of 3-manifolds. Invent. Math. 140, (2001) 45-100.

[3] T. Cochran, K. Orr and P. Teichner. Knot concordance, Whitney towers and $L^{2}$-signatures. Annals of Math. Vol. 157 No.2, (2003) 433-519.

[4] J. Conant, R. Schneiderman and P. Teichner. A geometric Jacobi relation in 3 and 4 dimensions. (In preparation.)

[5] J. Conant and P. Teichner. Grope coborbism of classical knots. (To appear in Topology), (2000). arXiv:math.GT/0012118

[6] J. Conant and P. Teichner. Grope coborbism and Feynman diagrams. (To appear in Math. Annalen.), (2002). arXiv:math.GT/0209075

[7] M. H. Freedman and F. Quinn. The topology of 4-manifolds. Princeton Math. Series 39, (1990).

[8] D. Gabai. Convergence groups are Fuchsian groups. Ann. of Math. Vol. 36, (1992) 447-510.

[9] S. Garoufalidis and J. Levine. Homology surgery and invariants of 3-manifolds. Geometry and Topology Vol. 5, (2001) 551-578. 
[10] C. H. Giffen. Link concordance implies link homotopy. Math. Scand. 45, (1979) $243-254$

[11] D. Goldsmith. Concordance implies homotopy for classical links in $M^{3}$. Comment. Math. Helvetici 54, (1979) 347-355.

[12] K. Habiro. Claspers and finite type invariants of links. Geometry and Topology Vol. 4, (2000) 1-834.

[13] A. Hatcher. Notes on basic 3-manifold topology. Posted at: ww. math. cornell. edu/ hatcher

[14] W. Jaco. Roots, relations and centralizers in 3-manifold groups. Lecture Notes in Math. Vol. 438, (1975) 283-309.

[15] W. Jaco and P. Shalen. Seifert fibered spaces in 3-manifolds. Memoirs of Amer. Math. Soc. Vol. 21 No. 220, (1979).

[16] W. Jaco and P. Shalen. A new decomposition theorem for irreducible sufficientlylarge 3-manifolds. Proceedings of Symposia in Pure Mathematics, Amer. Math. Soc. Vol. 32, (1978) 71-84.

[17] K. Johannson. Homotopy equivalences of 3-manifolds with boundary. Lecture notes in Math. Vol. 761, Springer-Verlag, (1978).

[18] E. Kalfagianni. Finite type invariants for knots in 3-manifolds. Topology 37, (1998) 673-707.

[19] P. Kirk and C. Livingston. Type 1 knot invariants in 3-manifolds. Pacific Journal of Mathematics Vol. 183 No. 2, (1998) 305-331.

[20] P. Kirk and C. Livingston. Knot invariants in 3-manifolds and essential tori. Pacific Journal of Mathematics Vol. 197 No. 1, (2001) 73-96.

[21] W. Magnus, A. Karass and D. Solitar. Combinatorial group theory: presentations of groups in terms of generators and relations. Dover Publications Inc., (1976).

[22] R. Schneiderman. Whitney towers and Gropes in 4-manifolds. Preprint (2003).

[23] R. Schneiderman. Simple Whitney towers, half-gropes and the Arf invariant of a knot. Preprint (2002).

[24] R. Schneiderman. Linking invariants and essential tori in 3-manifolds. (In preparation).

[25] R. Schneiderman. Stably slicing knots in 3-manifolds. (In preparation).

[26] R. Schneiderman and Peter Teichner. Higher order intersection numbers of 2spheres in 4-manifolds. Algebraic and Geometric Topology Vol. 1, (2001) 1-29.

[27] R. Schneiderman and Peter Teichner. Whitney towers, Milnor's invariants and the Kontsevich integral. (In preparation).

[28] C. T. C. Wall. Surgery on Compact Manifolds. London Math. Soc. Monographs 1, Academic Press, (1970) or Second Edition, edited by A. Ranicki, Math. Surveys and Monographs 69, A.M.S. 
[29] H. Whitney. The self intersections of a smooth $n$-manifold in $2 n$-space. Annals of Math. 45, (1944) 220-246.

Courant Institute of Mathematical Sciences

New York University

251 Mercer Street

New York NY 10012-1185, USA

Email: schneiderman@courant.nyu.edu

Received: 26 February 2003 Revised: 17 July and 2 September 2003

Algebraic 63 Geometric Topology, Volume 3 (2003) 